\title{
Mouse brain-wide mitochondrial connectivity anchored in gene, brain and behavior
}

\author{
Ayelet Rosenberg ${ }^{1 *}$, Manish Saggar ${ }^{2 *}$, Peter Rogu ${ }^{3}$, Aaron W. Limoges ${ }^{4}$, Carmen Sandi ${ }^{5}$, \\ Eugene V. Mosharov ${ }^{6}$, Dani Dumitriu ${ }^{3,7,8}$, Christoph Anacker ${ }^{3,4,8,9}$, Martin Picard ${ }^{1,9,10}$ \\ ${ }^{1}$ Division of Behavioral Medicine, Department of Psychiatry, Columbia University Irving Medical \\ Center, New York NY \\ ${ }^{2}$ Department of Psychiatry and Behavioral Sciences, Stanford University \\ ${ }^{3}$ The Sackler Institute, Department of Psychiatry, Columbia University Irving Medical Center, \\ New York NY \\ ${ }^{4}$ Division of Systems Neuroscience, Department of Psychiatry, Columbia University Irving \\ Medical Center, New York NY \\ ${ }^{5}$ Brain Mind Institute, EPFL, Switzerland \\ ${ }^{6}$ Division of Molecular Therapeutics, Department of Psychiatry, Columbia University Irving \\ Medical Center, New York NY \\ ${ }^{7}$ Department of Pediatrics, Columbia University Irving Medical Center, New York NY \\ ${ }^{8}$ Division of Developmental Neuroscience, Department of Psychiatry, Columbia University Irving \\ Medical Center, New York NY \\ ${ }^{9}$ New York State Psychiatric Institute, New York NY \\ ${ }^{10}$ Department of Neurology, Columbia University Irving Medical Center, New York NY
}

*These authors contributed equally to the work

Correspondence: martin.picard@columbia.edu 


\section{Abstract}

The brain and behavior are under energetic constraints, which are likely driven by mitochondrial energy production capacity. However, the mitochondria-behavior relationship has not been systematically studied on a brain-wide scale. Here we examine the association between mitochondrial health index and stress-related behaviors in mice with diverse mitochondrial and behavioral phenotypes. Miniaturized assays of mitochondrial respiratory chain function and mitochondrial DNA (mtDNA) content were deployed on 571 samples from 17 brain regions. We find specific patterns of mito-behavior associations that vary across brain regions and behaviors. Furthermore, multi-slice network analysis applied to our brain-wide mitochondrial dataset identified three large-scale networks of brain regions. A major network composed of cortico-striatal regions exhibits highest mitochondria-behavior correlations, suggesting that this mito-based network is functionally significant. Mito-based networks can also be recapitulated using correlated gene expression and structural connectome data, thereby providing convergent multimodal evidence of mitochondrial functional organization anchored in gene, brain and behavior.

Keywords: mitochondria, behavior, stress, connectivity, network analysis, mouse 


\section{Introduction}

The shaping of behaviors by life experiences is driven by energetically demanding circuitry across the brain (1). The brain's enormous energetic demand is mainly met by ATP produced through oxidative phosphorylation (OXPHOS) within mitochondria (2). As a result, mitochondria influence multiple aspects of brain development and function ranging from dendritic and axonal branching $(3,4)$, remodeling of gene expression (5), regulation of neurotransmitter release and excitability in mature synapses (6-8), neurogenesis (9), and inflammation (10). Thus, the mounting molecular and functional evidence that the brain is under energetic constraints $(11,12)$ suggests that a fundamental understanding of brain mitochondrial biology is essential for uncovering the basis of brain function and behavior.

Mitochondria are small, dynamic, multifunctional organelles with their own genome that generate ATP via the respiratory chain $(\mathrm{RC})$ (13). But not all mitochondria are created equal. Mitochondria subserving different cellular demands (i.e., in different cell types) have different relative molecular compositions and functional phenotypes (14-17). Therefore, developing a comprehensive understanding of the association between mitochondria and animal behavior calls for assessments of multiple mitochondrial features, across multiple brain regions. By analyzing mitochondrial features across multiple brain regions simultaneously, we can also potentially uncover unknown brain "mitochondrial circuits". This idea aligns with our evolving understanding of large-scale brain circuitry and metabolism $(11,18)$, and of the network distribution of neural activity achieved using brain-wide, high spatial resolution methods (e.g., MRI-based functional and structural connectivity maps) $(19,20)$. Although a similar degree of resolution for mitochondrial phenotyping is not feasible with current technologies, miniaturization of biochemical and molecular mitochondrial assays (21) open new possibilities to map mitochondria-to-behavior across multiple cortical and sub-cortical regions in the same animal. 
Both animal behaviors and mitochondrial health exhibit naturally occurring and acquired (i.e., experience-dependent) variation, providing an opportunity to map their associations. For example, behaviorally, exposure to social stress such as chronic social defeat predictably increases potential threat vigilance and social avoidance (22), but animals differ in their vulnerability/resilience to these stress-induced changes in behavior $(23,24)$. Experimental challenges aimed at modeling neuroendocrine disturbances resulting from chronic stress, such as chronic exposure to corticosterone (CORT), also induce avoidance behaviors associated with recalibrations in specific brain circuitry (25), gene expression $(26,27)$, and anatomical plasticity (i.e., atrophy) in stress-sensitive brain regions like the hippocampus (28). In relation to mitochondria, a separate body of literature similarly documents naturally-occurring mitochondrial variation $(21,29)$, as well as stress-induced functional recalibrations in mitochondria that occur within days to weeks of stress exposure (30-32) (meta-analysis in (33)), potentially linking mitochondrial health to behavior. More direct causal experiments show that mitochondrial RC function directly influence the brain and specific behavioral domains including working memory $(34,35)$, social dominance $(29,36,37)$ and anxiety-related behavior (4). Targeted mitochondrial defects even cause mood disorder-like phenotypes in animals (38) and are likely implicated in the etiology of psychiatric, neurological and degenerative disorders in humans (39). Moreover, in vivo human brain metabolic imaging studies show that energy metabolism in specific brain areas (e.g., nucleus accumbens, NAc) predict performance and anxiety (40-42). Thus, current evidence positions mitochondria as an upstream modulator of brain function and behavior.

Although the importance of mitochondria for brain structure and function is unequivocal, we lack an understanding of potential differences in mitochondria across different brain regions, therefore calling for a more complete mapping of region-specific brain mitochondrial function in relation to behavior. To address the hypothesis that mitochondrial health in specific brain regions is associated with behaviors, we have further miniaturized existing biochemical and 
molecular assays of mitochondrial RC function for sub-milligram tissue samples and deployed them across 17 cortical and sub-cortical brain regions in mice with a wide range of stressenhanced behavioral phenotypes, quantified through four behavioral tests. Using network-based connectivity analysis, we also sought to explore mitochondrial properties across brain regions, finding initial evidence that mouse brain mitochondria specialize as distinct functional networks linked to behavior. Further, we use gene co-expression and structural/anatomical connectivity data, which anchor the newly observed mitochondrial circuits into other modalities.

\section{Results}

Miniaturization of mitochondrial assays for sub-milligram resolution

Although enzymatic activity assays directly reflect the capacity of $\mathrm{RC}$ complexes and therefore energy production capacity, currently available assays suffer from low throughput that preclude a multi-region, brain-wide analysis in dozens of animals. Building from our previous efforts to scale throughput of mitochondrial biochemical assays (21), here we miniaturized and optimized assays for 96-well plate format to establish the lowest detection limit for mouse brain tissue. With this optimized platform, we can quantify the activities of $\mathrm{RC}$ complex I (CI, NADH dehydrogenase), complex II (CII, succinate dehydrogenase), and complex IV (CIV, cytochrome c oxidase) enzymatic activities, as well as the Krebs cycle enzyme citrate synthase (CS, a marker of mitochondrial content) in two $1 \mathrm{~mm}$-diameter, $200 \mu \mathrm{m}$ deep tissue punches. This represents $<1 \mathrm{mg}$ of tissue (estimated $0.33 \mathrm{mg}$ ), over an order of magnitude more sensitive than currently available methods.

In our pipeline, the same biological sample used for enzymatic activities is also used to quantify mtDNA abundance, both by i) the classical metric relative to the nuclear genome mtDNA/nDNA ratio, termed mtDNA copy number (mtDNAcn) (43), and ii) per tissue volume (mtDNA copies per mg), termed mtDNA density. From our qPCR data of nuclear genome abundance across the brain, independent of the mtDNA, we find that cellular density varies by 
up to 8.5-fold between brain regions (highest: cerebellum, lowest: visual cortex). Unlike in peripheral tissues, this remarkably large variation in neuronal and glial cells somata density significantly skews mtDNAcn estimates. Figure $\mathbf{S 1}$ shows the inter-correlations of mtDNAcn and mtDNA density in relation to enzyme activities, establishing that mtDNA density is a more appropriate and generalizable estimate of mitochondrial genome abundance across mouse brain regions.

We also combine these five primary mitochondrial features into a mitochondrial health index $(\mathrm{MHI})$ by dividing $\mathrm{RC}$ function $(\mathrm{Cl}+\mathrm{Cll}+\mathrm{CIV})$ by mitochondrial content (CS+mtDNA density), thus creating an index of energy production capacity on a per-mitochondrion basis (21) (See Methods for details). Although each of the six resulting features are partially correlated with other features, the proportion of shared variance between individual features across brain regions is $31-64 \%$, indicating that they each contribute some non-redundant information about mitochondrial phenotypes, which can be deployed at scale.

Our study design aimed to profile animal-to-animal differences in mitochondrial health across a broad set of brain regions that are associated with anxiety-related behavior, social behaviors, cognition, and mitochondrial disorders, and to relate these measures to each animal's behavioral phenotypes. In total, we enzymatically and molecularly phenotyped 571 samples covering 17 cortical, sub-cortical and brainstem brain regions isolated by bilateral punches at defined stereotaxic coordinates (Table 1, Figure S2B). To eventually compare the specificity of our findings related to brain and behavior, 5 peripheral tissues were also collected and analyzed from each animal, which we expected to show somewhat related but more modest associations with behavioral outcomes.

\section{Protein levels do not reflect enzymatic activity}

We initially explored if $R C$ protein abundance is a viable surrogate for mitochondrial $R C$ activity (29), which could theoretically allow high spatial resolution imaging of the entire brain. 
We focused on the cerebellum due to its well-defined cellular composition and laminar organization, where the Purkinje cell layer is flanked by molecular and granular layers (14) (Figure S3A). Compared to protein levels, enzymatic activity ultimately determines mitochondrial RC function and energy production capacity and consequently should be regarded as the most representative measure of mitochondrial function. In consecutive cerebellar slices, we compared SDH (CII) enzymatic activity to the protein abundance of a RC complex II subunit, SDHA (succinate dehydrogenase, subunit A), for which a validated highaffinity antibody is available. Enzyme activity did not correlate with protein abundance for any of the three cerebellar layers (proportion of shared variance, $r^{2}=0.02-0.07$ ), indicating that protein abundance is not an appropriate surrogate for enzymatic activity (Figure S3B). Reasons for this finding could include the action of post-translational modifications, stoichiometry of the four SDH subunits, or the biochemical context that drive activity independent of protein content (e.g., (44)). Therefore, we focus downstream analyses on direct measures of mitochondrial RC enzymatic activity and mtDNA density.

\section{Diversity of mitochondrial $R C$ activities between animals}

We sought to examine mitochondria-behavior associations in a cohort of adult male mice exhibiting naturally-occurring behavioral and mitochondrial variation. Our goal was to identify robust and generalizable associations between mitochondrial function and behavior, rather than potential correlations that would exist among only subgroups of animals (naïve or stressed). Therefore, to further extend the spectrum of mito-behavior variation using well-characterized rodent stress models, subgroups of the cohort were either chronically administered corticosterone (CORT) (45), or exposed to chronic social defeat stress (CSDS) (46) (Figure S2A). To create further diversity among groups, half of the CSDS mice were allowed to recover for two months. Based on previous work $(23,47)$, we also expected naturally-occurring behavioral and brain molecular phenotype differences between animals that are resilient or 
susceptible to CSDS (based on the social interaction test; see Methods) (48). The naturally occurring variation in mitochondria and behavior plus the effects of various exposures provides a strong test of robustness for our hypothesis, which is that across a diverse population of mice, mitochondrial health in specific brain regions are consistently associated with behaviors.

Across our cohort of inbred mice with a range of exposures, a wide range of mitochondrial health was observed. The average variation for mitochondrial activities (excluding $\mathrm{MHI}$ ), across all animals and all 17 brain regions was 36\% (coefficient of variation, C.V.) (Figure S4B). For any given brain region, the variation in mitochondrial health between mice reached up to 2.9-fold between the animals with the lowest and highest activities. This means that for a given brain region, there are large mouse-to-mouse differences in mitochondrial content and RC activity, even among inbred control mice not exposed to stressors (Figure S4). Relative to brain regions, peripheral tissues showed about a third less variation between animals, with an average 25\% C.V. (Figure S4D).

To verify that interventions used to enhance naturally-occurring variation in mitochondria were effective, we compared mitochondrial features in mice exposed to CORT and CSDS relative to naïve mice. The effects of CORT and CSDS on mitochondrial health were quantified as standardized effect sizes (Hedges g) and shown in (Figure 1A, detailed in Figure S5). Both interventions significantly altered mitochondrial features in a region-specific manner. CORTtreated mice had higher mitochondrial activities than non-stressed animals in $\sim 60 \%$ of brain regions, whereas CSDS animals had lower activities in $\sim 82 \%$ of brain regions (Figure 1D), suggesting opposing effects of these two different stress models on brain mitochondria. The amygdala showed the greatest CORT-induced increase in Cll enzymatic activity (+49\%, $\mathrm{p}<0.05)$, whereas the periaqueductal gray $(P A G)$ showed the largest CSDS-induced decrease in $\mathrm{Cl}$ activity $(-42 \%, \mathrm{p}<0.05)$. 
In order to determine if brain regions were co-regulated in their stress-induced mitochondrial recalibrations, we employed a topological data analysis (TDA)-based Mapper approach. Mapper is a variant of nonlinear dimensionality reduction (manifold learning) methods that produces a graph or network embedding of the high-dimensional data (a.k.a. shape graph) while recovering projection loss using an additional partial clustering step $(49,50)$. When applied to mitochondrial features, separately for each group, the shape graphs revealed differences in regional mitochondrial recalibrations across the two groups (Figure 1E-F). This was quantified using a graph-theoretical measure of participation coefficient (PC) (51), where higher values of PC indicate uniformly distributed connectivity across regions (integrated network) and lower values indicate a segregated connectivity pattern (Figure 1G). CORTinduced mitochondrial recalibrations were relatively more region-specific (or segregated) whereas CSDS caused a more coherent response across the brain (or integrated).

Although we were not powered to make statistical comparisons between susceptible and resilient mice, we did observe relatively large differences in effect sizes $(g>0.8)$ in mitochondrial recalibrations between the groups (Figure S6). Additionally, compared to nonrecovered CSDS mice, animals who were allowed to recover from CSDS exhibited only marginal mitochondrial health changes in a limited number of brain regions, as visualized by the Mapper-generated shape graphs (Figure S7). Overall, these results confirm the existence of naturally-occurring and acquired variation in brain mitochondrial health between animals, providing a strong basis to test the existence of conserved associations with behaviors.

\section{Diversity of anxiety and depressive like behaviors}

Just as there was a spectrum of mitochondrial health across mice, as expected from previous work, animals also naturally exhibited large variation in their behavioral phenotypes. Behavioral tests included potential threat ("anxiety") monitored by the open field test (OFT) and elevated plus maze (EPM), hyponeophagia monitored by novelty suppressed feeding test 
(NSF), and approach-avoidance conflict using the social interaction test (SI). Because specific behavioral tests are generally administered in conjunction with specific interventions (CORT, CSDS), some animals were only tested on some and not all behaviors. Both CORT and CSDS produced the expected elevation in anxiety-related behavior compared to naïve mice (Figure S8). Results from the behavioral tests either correlated moderately with each other (EPM and OFT, $r=0.60$ ), were not correlated (OFT and NSF, $r=-0.02$ ), or were negatively correlated (OFT and SI, $r=-0.46)$. This indicated that each test captures different aspects of behavior, thereby providing a robust basis to examine how different aspects of behavior might relate to brain mitochondrial health.

\section{Brain mitochondrial health correlates with specific behaviors}

We next evaluated the extent to which mitochondrial health in different brain regions was associated with behavior (Figure 2A). Behavioral scores were transformed so that higher scores on each test indicate higher avoidance/anxiety-like behaviors (as in (23)) (See Methods, Figure S8). Behavioral scores were then correlated with mitochondrial function across the 6 mitochondrial features, where higher values indicate higher mitochondrial content or functioning. A frequency distribution of the effect sizes for all mitochondrial-behavior pairs revealed a significant non-zero correlation between $\mathrm{MHI}$ in the 17 brain regions and behavior on OFT $(p<0.01)$, EPM $(p<0.0001)$, and SI $(p<0.0001)$, but not for NSF (Figure 2B, frequency distributions for the other mitochondrial features are shown in Figure S9). Time to feed on the NSF was capped at 600 seconds, so the correlations are less precise than for other tests. To better understand which regions were driving the direction and magnitude in the distributions, we then examined the patterns of correlations for all 17 brain regions, for all six mitochondrial features, across the four behavioral tests (Figure 2C).

For both OFT and EPM, higher mitochondrial health in the majority of brain regions was correlated with higher avoidance scores (average $r=0.12,0.34$ respectively). The highest 
correlations were of 0.51 for M1 CII and OFT ( $p=0.025$, linear regression), and 0.92 for NAc $\mathrm{MHI}$ and EPM $(\mathrm{p}=0.0005)$. For NSF there was more heterogeneity between brain regions, with a similar number of positive and negative correlations. Finally, for SI, we observed the opposite relationship; animals with higher mitochondrial functioning generally displayed greater sociability (lower social avoidance) (average $r=-0.21$, highest for $\mathrm{MHI}$ in $\mathrm{SN}$ and $\mathrm{SI}, \mathrm{r}=-0.78, \mathrm{p}=0.0035$ ). Interestingly, of the six mitochondrial features, the strongest mito-behavior correlations (for 3 out of 4 behaviors) were for $\mathrm{MHI}$, which may reflect the superiority of $\mathrm{MHI}$ as an integrative measure of mitochondrial health over individual enzymatic and molecular features.

As expected, the average correlation between mitochondrial function and behaviors was significantly more consistent for brain mitochondria than for mitochondria in peripheral tissues (Figure 2D). This finding has two implications. First, it reinforces the specificity of these mitobehavior findings for the brain. Second, it implies that mitochondria across the brain and other tissues within an individual mouse are not equivalent, and differentially regulated. This naturally raised the question whether specific brain regions within an animal could also exhibit independently regulated mitochondrial properties. In other words, whether there exist a functional organization of the brain into separate networks based on mitochondrial properties.

\section{Mitochondrial function-based organization of the brain}

To address this question, we first asked whether mitochondrial features in each tissue were independent or correlated with other tissues. Using the same rationale that underlie functional connectivity analysis in fMRI data (52-54), we first generated a correlation-based similarity matrix of all mitochondrial features across examined brain regions and non-brain tissues (Figure 3A). Within the 17 brain regions, mitochondrial features were generally positively correlated (average $r=0.22, p<1^{-100}$, two sample t-test), with a few exceptions. Higher mitochondrial activities in one brain region generally also implies higher activities in other regions $\left(\mathrm{p}<1^{-82}, 1\right.$-sample t-test). Further, a modular structure was also apparent, within brain 
and peripheral regions, such that mitochondrial features within each region was more similar than with others regions $(p<0.0001$, permutation test); Figure $3 \mathbf{B}$ also shows the average correlations of each mitochondrial feature with other features among all brain regions - RC enzymes were most strongly co-regulated. To compute how similar each region is to other regions, we computed nodal degree (i.e., average inter-regional correlation) for all regions. Nodal degree, or global connectivity, was highest in the cerebellum (average $r=0.34$ ) and lowest in the brainstem vestibular nucleus $(r=0.05)$ (Figure $3 \mathbf{C})$.

As suggested above from the divergence between brain regions and peripheral tissues in the mito-behavior correlation patterns, the mitochondrial features in peripheral tissues were not correlated with brain mitochondria (average $r=0.02$ ) or with other peripheral tissues mitochondria (average $r=-0.03$ ) (Figure 3D). The lack of association between brain and nonbrain tissues of the same animals suggest that $\mathrm{MHI}$ (as well as content and $\mathrm{RC}$ activities) is not a ubiquitous animal-level feature, but rather relatively independently defined in the brain, and further specified within different brain regions.

Given the positive connectivity across the brain and recent evidence of circuit-level metabolic coupling in the Drosophila brain (12), we examined whether certain regions exhibited particularly strong mitochondrial feature co-regulation. Functional brain networks in the living brain are defined by synchronous activity patterns (e.g., default-mode network, fronto-parietal network). Similarly, we reasoned that connectivity patterns of mitochondrial features (based on single measurements) could reflect networks of brain regions with similar bioenergetic properties. To examine this hypothesis with a framework agnostic to anatomical categorization and inclusive of all mitochondrial features, we performed multi-slice community detection analysis (55), with mitochondrial features represented in six separate layers (Figure 3E). Categorical multi-slice community detection allowed for detecting cohesive groups of brain regions, or communities, that: i) are more similar to each other than they are to the rest of the 
regions, and ii) have cohesion converging across the six layers of mitochondrial features. Representing all 17 brain regions, and across 6 mitochondrial features, we found 3 separate communities, or networks: 1) Cortico-striatal network: CPu, visual, motor, mOFC, mPFC and NAc; 2) Salience/Spatial navigation network: cerebellum, vestibular nuclei, VTA, thalamus, hippocampus (CA3), and dentate gyrus (dorsal and ventral); and 3) Threat response network: amygdala, hypothalamus, substantia nigra, and periaqueductal grey (Figure 3F).

To examine whether this functional organization of the brain revealed using mitochondrial features is also evident cross-modally, we used Allen Brain Atlas datasets of brain-wide gene co-expression (56) as well as EYFP-labeled axonal projections that define the structural connectome (57) in the same mouse strain. Specifically, we examined whether gene expression correlations and structural connectivity within brain regions that are functionally grouped together as a network, based on mitochondrial features, is higher than expected by chance. We used two independent graph theoretical metrics: strength fraction (S.F.) (58) and quality of modularity (Q_mod) (59), to examine whether similar communities exist in gene and structural connectivity data. Using permutation testing, we randomly shuffled community structure of brain regions 10,000 times to determine whether the community structure derived from mitochondrial features is also evident in gene co-expression and structural connectivity data. Both strength fraction and quality modularity statistics suggest that mitochondria-derived networks also have higher similarity in gene co-expression and structural connectome data than expected by chance (S.F.-gene-coexpression, $p=0.020$; S.F.-structural connectivity, $p=0.029$; Q_mod-gene-coexpression, $p=0.008$ ); and $Q \_m o d-s t r u c t u r a l$ connectivity, $p=0.015$ ) (Figure S10). Hence, this provides convergent multimodal evidence of mitochondrial functional organization overlapping with gene expression and the structural connectome. 
Finally, to understand the potential significance of this effective brain-wide mitochondrial connectivity in relation to animal behavior, we used our mitochondria-derived network results to partition the brain into three networks, and then analyzed the ability of each network to linearly predict behaviors. The mitochondrial-behavior correlations varied both in strength and direction between the three networks. The average mitochondrial activity in the cortico-striatal network consistently showed the strongest average correlations with behaviors measured in the OFT, EPM, and SI tests $(r=0.20,0.58,-0.41$, respectively), whereas the spatial navigation network exhibited the weakest average correlations for the same three behaviors $(r=-0.03,0.18,-0.14)$

(Figure 3G). Together, these results suggest the presence and functional significance of brainwide mitochondrial networks, embedded within existing neural circuitry in the mouse brain.

\section{Discussion}

Using a high-throughput approach to functionally phenotype hundreds of brain samples from a heterogenous mouse cohort, we have defined brain-wide associations between mitochondrial health and behaviors. Combined with previous findings $(29,32)$, the diverging mito-behavior associations between brain regions, and between brain and non-brain tissues, brought to light the possibility that different brain regions might exhibit different mitochondrial phenotypes. Examining the network characteristics of mitochondrial features across the brain provides evidence for the modular distribution of mitochondria across cortical and sub-cortical regions, and their relevance to depression-like and anxiety-like behaviors. Based on these data, we conclude that mouse brain mitochondria may exist as behaviorally-relevant networks overlapping with, but distinct from, other modalities such as gene expression and structural connectivity.

Utilizing CORT and CSDS interventions to induce behavioral variation in the population also allowed us to compare the effects of these two interventions on mitochondrial functioning. 
Different brain regions were found to respond, in some cases, in opposite directions, particularly after exposure to CORT. This could be related, among other factors, to regional differences in glucocorticoid and mineralocorticoid receptor density. In contrast to CORT, the recalibrations of brain mitochondria to CSDS was more uniform, with the majority of brain regions exhibiting a coordinated reduction in most mitochondrial features. These results highlight a significant difference between the effects of behavioral stress and endocrine interventions on mouse brain bioenergetics, which should be taken into account when selecting and interpreting rodent models of chronic stress.

Previous research in rodents showed that mitochondrial function in specific brain regions, such as the NAc, is linked to complex behaviors such as social dominance and anxiety $(4,29)$. Here we confirm the strong correlation between NAc mitochondrial health and anxious behavior, as the strongest correlation between EPM-based anxiety-like behavior and $\mathrm{MHI}$ is in the NAc. We also extend this finding to show that mitochondrial health is also linked to behaviors across distributed brain networks. Similar to the conceptual shift from regional and cellular perspectives towards distributed brain networks and neuronal ensembles $(60,61)$, our discovery advances the notion that mitochondria may regulate brain function and behavior through distributed mito-networks. This notion is consistent with recent evidence of metabolic coupling among distributed patterns of behavior-related neural activity in Drosophila (12).

While we cannot directly explain the apparent harmonization of mitochondrial function across brain regions, it may arise from three main factors. First, mitochondria could respond to differences in neuronal circuit functioning, in agreement with observed coupling of neuronal and metabolic activities (12), such that the cellular infrastructure of neural circuits that fire together not only wire together, but also generate similar mitochondria. A second possibility is that brain regions which are regularly co-activated, i.e., within the same functional networks, harbor similar levels of receptors for neuroendocrine factors (stress and sex steroids) known to influence 
mitochondrial biogenesis and/or functional specialization $(62,63)$. A third possibility would involve differences in metabolites and substrates that contribute to mitochondrial functioning and regulation, arising similarly among co-activated brain regions. These and other potential factors underlying the modularity of mitochondria within the mouse brain remain to be investigated.

From a mitochondrial biology perspective, our data highlights a potentially important distinction between mitochondrial content (abundance of mitochondria) and RC activity normalized to mitochondrial content, such as the MHI (respiratory chain activity per mitochondrion). These frozen tissue measurements naturally reflect the maximal functional capacity of the mitochondria $\mathrm{RC}$, rather than their actual in vivo rates, which are likely driven by neural activity. Interestingly, the behavioral correlations with content features (mtDNA density and CS activity) for social avoidance behavior were similar to one another, and differed somewhat from RC enzymes. Experimentally, the specificity of these findings highlights the value of parallel assessments of multiple mitochondrial features reflecting unique mitochondrial properties (i.e., mitochondria are not all created equal). In neuroimaging terms, the composite $\mathrm{MHI}$ can be understood as the mitochondrial analog to $\mathrm{fMRI}$-based multi-variate pattern analysis (MVPA) $(64,65)$, where multiple features (mitochondrial enzymes for MHI, voxels for MVPA) are combined to create a more stable and statistically accurate metric of the desired outcome (mitochondrial health for MHI, or brain activation for MVPA). Moreover, mtDNAcn has previously been assessed across multiple brain regions (66), but our results here go beyond in showing that quantifying mtDNA content on a per-cell basis (mtDNA:nDNA ratio) is heavily skewed by cellularity. As such, it is driven by how many cell bodies are present in the tissue, and correlates poorly with both mitochondrial content and RC activity. Therefore, our data reinforce the notion that mtDNAcn on its own is not a valid measure of mitochondrial health $(67,68)$. In the mouse 
brain, our findings shows that mtDNA density per unit of volume (rather than per cell) is a more biologically meaningful mitochondrial feature.

By examining mitochondrial features across the mouse brain, we discovered a moderate level of global functional connectivity across mitochondria in most brain regions, but not among peripheral tissues (Figure 3). By functional connectivity, we do not imply that mitochondria are directly connected to each other in the same way that neurons project and chemically (de)activate each other; but rather than they share functional properties. As a biological property of the system, if mitochondrial function was directly determined by genetic and physiological factors, the logical expectation is that mitochondria within different organs of the same organism should exhibit a high degree of coherence (i.e., correlated with each other). In other words, the animal with the highest mitochondrial content or RC activity in the mOFC should also be one of the highest in other brain regions and tissues. But our results strongly disprove this point. Similarly, previous work on multiple human tissues showed that mtDNAcn was not significantly correlated between organs, including across three brain regions (69). Here we extend these data to 17 brain regions and 5 non-brain tissues, demonstrating that identified correlations are relatively modest (the highest is $r=0.31$ for the cerebellum, or less than $10 \%$ of shared variance). Some brain regions exhibited no correlation with other regions. The most parsimonious explanation is that animals differentially recruit different circuitry, which secondarily shape their mitochondria. However, considering theories that stipulate that the brain strives for maximal energetic efficiency (1), we also cannot exclude the possibility that a limited quantity of resources (i.e., mitochondrial function) is available within the brain or the whole organism, which are distributed unequally in an activity-dependent manner among different organ systems, functional networks, and individual brain regions. Thus, different animals may achieve an optimal balance of systemic and neural function through specific combinations of mitochondrial activity in different brain regions, a possibility that remains to be tested. 
In trying to better understand why certain regions displayed stronger brain-wide connectivity than others, we discovered mitochondria-based communities, or networks of brain regions. Interestingly, we observe some parallels in our mitochondria-derived networks with established large-scale networks. For example, the cortico-striatal network identified includes the $\mathrm{CPu}, \mathrm{NAc}, \mathrm{mOFC}, \mathrm{mPFC}$, and motor and visual cortex, which are implicated in decision making and executing actions. The second identified network (Cereb, VN, VTA, Thal, CA3, DGv and DGd) is the most heterogenous but comprised of regions that are known to be connected and involved in salience and spatial navigation (70). Lastly, the final circuit is made up of limbic and limbic-associated regions (Amyg, Hypoth, PAG and SN) involved in threat responses (71, 72). In comparing these mitochondria-derived networks to gene expression and structural connectome data we found that the communities significantly overlap across modalities, but are not identical. Finally, each network's mitochondrial health exhibited different associations with behavioral responses. Unsurprisingly, the cortico-striatal network explained the greatest proportion of variance in behavior, increasing the likelihood that the identified large-sclae mitochondrial networks are functionally relevant. In our view, this novel perspective should provide a basis to further delineate the functional brain-wide organization of mitochondria.

Until now, the methods used to reveal neurobiological and metabolic networks in mammals have typically been through indirect functional and structural connectivity analysis (18). Here we have developed a scalable approach to examine bioenergetic, and more specifically mitochondrial features across a large number of brain regions in mice with predefined behavioral phenotypes. Further, we showed that bioenergetic connectivity was tightly linked with gene co-expression and structural connectivity, thereby providing converging evidence of mitochondria-based networks across modalities. This study synergizes with recent work in Drosophila (12), providing the technical and empirical foundation to bring mitochondrial biology into brain-wide, network-based models of neural systems in mammals (19, 20). Applied 
bioRxiv preprint doi: https://doi.org/10.1101/2021.06.02.446767; this version posted June 3, 2021. The copyright holder for this preprint (which was not certified by peer review) is the author/funder, who has granted bioRxiv a license to display the preprint in perpetuity. It is made available under aCC-BY 4.0 International license.

to mitochondria, network-based analytics will contribute to develop more accurate maps, and eventually an understanding of what determines mitochondrial and metabolic properties across interacting neural circuits. Developing a spatially-resolved understanding of brain mitochondrial biology in diverse populations of animals and humans should help to resolve the energetic basis of brain function and behavior. 


\section{Methods}

\section{Animals}

This study was carried out in accordance with NIH Guidelines, and was approved by the Institutional Animal Care and Use Committee (IACUC) at New York State Psychiatric Institute. Adult (52 weeks old) C57BL/6J male mice were obtained from Jackson Laboratories $(\mathrm{n}=29)$. Three month-old CD1 retired breeder male mice were obtained from Charles River, and were used as the aggressors in the social defeat model.

\section{Chronic Social Defeat Stress (CSDS)}

Aggressors Prescreening: All CD1 mice used in the experiment were pre-screened for aggressive behaviors as previously described (47). During a three-day screening procedure, a novel C57BL/6J mouse was placed in the cage of the CD1 mouse for $3 \mathrm{~min}$. C57BL/6J screener mice were not further used in the study. The latency of the CD1 mouse to attack the C57BL/6J screener mouse was recorded. CD1 mice that attacked in less than 1 minute on at least the last two consecutive screening days were considered to be aggressive.

Experimental Groups: Thirteen C57BL/6J mice underwent social defeat stress, while 6 remained in their cages, to serve as the control group. Animals were randomly assigned to these groups. One mouse subjected to social defeat died of unknown causes during the duration of the experiment. Of the remaining 12 social defeat mice, half $(n=6)$ were sacrificed two days after the completion of the stressor, and they are referred to as the 'stressed' CSDS group. Three control mice were sacrificed at the same time. The other half of the CSDS mice $(n=6)$ along with 3 control mice, were allowed an 8.5 weeks (59 days) stress recovery period prior to sacrifice. This group is referred to as the 'recovered' group. Animals were selected for the recovery group from the CSDS group so that there was an even ratio of susceptible to resilient mice in the 'stressed" and 'recovered' groups. 
Social Defeat Paradigm: Adult experimental male C57BL/6J mice $(n=13)$ were exposed to a CSDS paradigm daily, for 10 days. The experimental C57BL/6J mice were placed in the cage of a new CD1 aggressor mouse for 5 minutes every day, for 10 consecutive days. After the 5 minutes of physical defeat, the $C 57 \mathrm{BL} / 6 \mathrm{~J}$ mice were housed in the same cage as the CD1 aggressor, with a perforated plexiglass divider to separate them for 24 hours. After the 10 days of defeats, experimental C57BL/6J mice and CD1 mice were singly housed. Adult control male C57BL/6J mice $(n=6)$ were housed 2 mice per cage separated by a perforated plexiglass divider. To control for the effects of experimental handling, each control mouse was paired with a new control mouse every day for 10 days.

\section{Corticosterone administration (CORT)}

Corticosterone (Sigma Aldrich, St Louis, MO) was dissolved in vehicle $(0.45 \%$ Bcyclodextrin) at a concentration of $35 \mu \mathrm{g} / \mathrm{ml}$, equivalent to administration of $\sim 5 \mathrm{mg} / \mathrm{kg} / \mathrm{day}$ per mouse (45). C57BL/6J mice $(n=5)$ were group-housed and administered corticosterone in their drinking water. Control mice $(n=5)$ were group-housed and received only vehicle. Animals were randomly allocated to the two groups. Water bottles were prepared twice a week, and the mice never had access to other water. Behavioral testing began on day 56 of CORT administration. Experimental and control mice were sacrificed on day 63 following the completion of behavioral testing. The duration of administration was 9 consecutive weeks.

\section{Behavioral tests}

Social Interaction (SI) Test: Social avoidance was measured $24 \mathrm{hrs}$ after the completion of the last day of defeat (day 11). During the first trial, experimental mice were allowed to explore an open field arena $(40 \mathrm{~cm} \times 40 \mathrm{~cm} \times 40 \mathrm{~cm})$ containing an empty wire enclosure for 2.5 minutes. During the second trial, a CD1 mouse was placed into the wire enclosure, and the C57BL/J6 mouse was reintroduced for 2.5 minutes. Time spent in the SI zone and time spent in the corner zones during the first and second trial were recorded. SI ratios were calculated as 
time spent in the SI zone during the second trial divided by time spent in the interaction zone during the first trial (23). Mice with SI ratios of $<1$ were considered "susceptible" $(n=6)$, while mice with SI ratios $>1$ were considered "resilient" $(n=7)(73)$. Corner zone ratios were calculated as time spent in the corner zones during the second trial divided by time spent in the corner zones during the first trial.

Open Field Test (OFT)- both groups: OFT were run 24 hrs after the SI tests for CSDS mice, and on day 56 of CORT administration. Each mouse was placed in an open field arena $(40 \mathrm{~cm} \times 40 \mathrm{~cm} \times 40 \mathrm{~cm})$ for 10 minutes. A camera on a tripod stand was set up above the arena to record the activity, and the video was later analyzed using Ethovision XT (Noldus). The percent of time spent in the center of the open field $(20 \mathrm{~cm} \times 20 \mathrm{~cm})$ and the percent distance traveled in the center of the open field were analyzed.

Elevated Plus Maze (EPM): EPM tests were done the day after the OFT for CORT mice (day 57 of treatment). Each mouse was placed in an elevated plus maze for 10 minutes. A camera on a tripod stand was set up above the arena to record the activity, and the video was later analyzed using Ethovision XT (Noldus). The percent of time spent in the open arms was analyzed.

Novelty Suppressed Feeding (NSF): NSF was performed after EPM for CORT mice (day 59 of treatment), and as previously described (74). Briefly, NSF testing apparatus consisted of a plastic box $(50 \times 50 \times 20 \mathrm{~cm})$ with $2 \mathrm{~cm}$ of wood chip bedding. The center of the arena was brightly lit (1200 lux). Mice were food restricted for $15 \mathrm{~h}$ during the dark phase prior to testing. At the time of testing, a single pellet of food (regular chow) was placed on a white paper platform positioned in the center of the box. Each animal was placed in a corner of the box, and a stopwatch was immediately started. The latency of the mice to begin eating was recorded during a 10-min test. Immediately after mice took a bite from the food pellet, the pellet was removed from the arena. Mice were then placed back in their home cage and latency to eat 
and the amount of food consumed in 5 min were measured (home cage consumption). NSF latency was capped at $10 \mathrm{~min}$, with animals that did not consume any portion of the pellet receiving a score of $600 \mathrm{sec}$.

Behavioral Z-Scores: Social avoidance scores were determined by averaging the $\mathrm{z}$ scores for 4 measures as previously described (23); SI ratio, corner ratio, time spent in SI zone, and time spent in corner zones. The $\mathrm{z}$ scores for SI ratio and time spent in SI zone were multiplied by -1 , so that higher scores across all 4 measures indicated higher avoidance. Similarly, the OFT score was determined by averaging the $z$ scores for the 2 measures. The $z$ scores were multiplied by -1 , so that higher OFT score indicate higher avoidance of the brightly lit center of the OF. EPM scores were also multiplied by -1 , so that higher EPM scores indicate higher avoidance of the open arms of the EPM. High NSF scores already indicate higher avoidance, so they were not inverted. Therefore, across all 4 tests, higher scores indicate higher anxiety-like/avoidant behavior.

\section{Tissue Collection}

Animals were sacrificed by rapid decapitation to maintain mitochondrial integrity in brain tissue. Brains were rapidly flash frozen in ice-cold isopentane, stored at $-80^{\circ} \mathrm{C}$, and later transferred to $-170^{\circ} \mathrm{C}$ (liquid nitrogen vapor) until mitochondrial measures were performed. Brains were transferred back to $-80^{\circ} \mathrm{C}$ during the week prior to being sectioned and were then transferred to $-30^{\circ} \mathrm{C}$ the night before sectioning. Brains were sectioned coronally on a Leica Model CM3050 S cryostat. The cryostat internal temperature and blade temperature were set to $-22^{\circ} \mathrm{C}$ during sectioning. Brains were mounted onto a specimen disk using optimal cutting temperature (OCT) compound. The brain was sectioned coronally, alternating between two $200 \mu \mathrm{m}$ thick slices and then two $20 \mu \mathrm{m}$, and were deposited onto microscope slides. Brain sections were kept at $-80^{\circ} \mathrm{C}$ until brain region-specific tissue sample collection. One of the 
recovered CDSD mouse brain contained blood and could not be reliably sectioned, so this animal was excluded from analysis. A second CSDS mouse brain and a control brain both cracked during slicing, so some of the brain regions ( $n=7$ for CSDS mouse, $n=8$ for control) could not be obtained from those brains, but the regions that were obtained were included in analysis.

Tissue biopsy punches on frozen brain sections: The scalable Allen Mouse Brain volumetric atlas 2012 (75) was used to determine the location of each brain region of interest, and their distance from bregma. The atlas' Nissl-stained images were used as a reference for estimating each section's distance from bregma, which determined which brain slices would be used for punch biopsy collection of each region of interest (Figure S2B). Brain slices were punched using $1.00 \mathrm{~mm}$ diameter Robbins True-Cut Disposable Biopsy. Two bilateral punches were collected for each brain region over dry ice. For brain regions in the midline, punches were taken from two consecutive slices. All tissue punch locations were approximated by using landmarks on the slice and by comparing to the atlas. It is important to note that the $1 \mathrm{~mm}$ puncher was larger than the actual brain region in some instances, and so parts of neighboring brain regions may have been included in the punches. Therefore, the punching technique may include some error. Punches were stored at $-80^{\circ} \mathrm{C}$ until they were ready to be used for enzymatic activity assays.

The tissue punches were too light to be accurately weighed, so we had to estimate the weight based on the reported mouse brain density of $1.04 \mathrm{~g} / \mathrm{cm}^{3}(76)$. The punches were $0.5 \mathrm{~mm}$ (diameter) by $200 \mu m$ (height), so using the equations $v=\pi r^{2} * h$ and $d=m / v$ we obtain an estimated mass of $0.1633 \mathrm{mg}$ per punch, thus 2 punches were approximated to weigh $0.3266 \mathrm{mg}$.

\section{Mitochondrial Measurements}


Tissue Preparation: The punches from each brain region were homogenized in $0.2 \mathrm{ml}$ of homogenization buffer (1mM EDTA and 50mM Triethanolamine) (2x 1mm tissue punch/ $0.2 \mathrm{~mL}$ of homogenization buffer), with 2 tungsten beads to disrupt the tissues' cells and release the mitochondria. Tissues were homogenized using a Tissue Lyser (Qiagen cat\# 85300), which was run at 30 cycles/sec for 1 minute. The tissues were then incubated in ice for 5 minutes, and were then re-homogenized for 1 minute. Tissues were vortexed to ensure homogeneity. Peripheral tissues were cut over dry ice, weighed, and were then homogenized 1:180 (weight:volume, mg: $\mu \mathrm{l}$ ), except for heart samples that were further diluted to $1: 720$ to be in the dynamic range of the assays.

\section{Enzymatic activities}

Enzymatic activities were quantified spectrophotometrically for Citrate Synthase (CS), Complex I, Succinate Dehydrogenase (SDH, complex II), Cytochrome C Oxidase (COX, complex IV) and and were expressed per mg of tissue, as described previously with some modifications (21). All miniaturized assay measurements were performed in 96-well plates and enzymatic activity assays recorded on a Spectramax M2 (Spectramax Pro 6, Molecular Devices). Linear slopes reflecting changes in absorbance of the reporter dye were exported to Microsoft excel and converted into enzymatic activities using the molar extinction coefficient and dilution factor for each assay. The assays were optimized to determine the minimal amount of tissue required to obtain reliable results assessed by the C.V. between duplicates. The assays were then further optimized to determine the minimal amount of brain tissue homogenate required for each individual assay. Assay validation involved regressing increasing tissue amounts (number of punches) with observed activities, which confirmed that an increase or decrease in tissue used produced proportional changes in total activity.

Samples were run in duplicates for each enzyme, along with a nonspecific activity control, and every plate had a positive control (heart homogenate). The 96-well plates were 
designed so that each brain region/tissue from all animals were run on a single plate, which prevents potential batch variation for comparisons between the animals. However, due to the size of the plates, no more than 2 types of tissues could be run together on a single plate. This limits the accuracy of the comparisons of enzymatic activities between tissues, but maximizes the accuracy of comparisons between animals for each tissue. Reference positive controls on each plate were used to control for potential batches/plates effects. None of the enzymatic activities of some samples ( $n=8$ out of 579 , resulting in $n=571$ ) could not be measured for technical reasons and are therefore not included in the analyses.

Citrate synthase (CS) enzymatic activity was determined by measuring the increase in absorbance of DTNB at $412 \mathrm{~nm}$ at $-30^{\circ} \mathrm{C}$ in $200 \mathrm{uL}$ of a reaction buffer (200 mM Tris, $\mathrm{pH} 7.4$ ) containing acetyl-CoA $10 \mathrm{mM}, 10 \mathrm{mM}$ 5,5'- dithiobis- (2-nitrobenzoic acid) (DTNB), $2 \mathrm{mM}$ oxaloacetic acid, and $10 \% \mathrm{w} / \mathrm{v}$ Triton-x-100. The rate of conversion of DTNB into NTB ${ }^{2-}$ ions indicates the enzymatic activity and is used as a marker of mitochondrial content. Oxaloacetate is removed from the assay mix as a way to measure non-specific activity. The final CS activity was determined by integrating OD412 change over 150-400 seconds and then subtracting the non-specific activity. $10 \mu \mathrm{L}$ of homogenate was used to measure CS activity.

Complex I (Cl) activity was determined by measuring the decrease in absorbance of DCIP. The rate of absorbance of DCIP is measured at $600 \mathrm{~nm}$ at $30^{\circ} \mathrm{C}$, in $200 \mu \mathrm{L}$ of a reaction buffer (potassium phosphate $100 \mathrm{mM}$, pH 7.4) containing $550 \mathrm{mg} / \mathrm{ml}$ bovine serum albumin (BSA), 50mM potassium cyanide (KCN), $20 \mathrm{mM}$ decylubiquinone, and $0.4 \mathrm{mM}$ antimycin $\mathrm{A}$. $10 \mu \mathrm{L}$ of homogenate was used to measure $\mathrm{Cl}$ activity. Antimycin $\mathrm{A}$ and $\mathrm{KCN}$ are used to inhibit electron flow through complexes III and IV. The negative control condition includes rotenone (200mm) and piericidin $\mathrm{A}(0.2 \mathrm{mM})$, which inhibit $\mathrm{Cl}$ activity. The final $\mathrm{Cl}$ activity was determined by integrating OD600 change over 150-500 seconds, and by subtracting the rate of NADH oxidation in the presence of rotenone and piericidin $\mathrm{A}$ from the total decrease in absorbance. 
$\mathrm{SDH}(\mathrm{CII})$ activity was determined by measuring the decrease in absorbance of DCIP. The rate of absorbance of DCIP was measured at $600 \mathrm{~nm}$ at $30^{\circ} \mathrm{C}$, in $200 \mu \mathrm{L}$ of a reaction buffer (potassium phosphate $100 \mathrm{mM}, \mathrm{pH} 7.4$ ) containing $50 \mathrm{mg} / \mathrm{ml}$ bovine serum albumin (BSA), $500 \mu \mathrm{M}$ rotenone, $500 \mathrm{mM}$ succinate-tris, $50 \mathrm{mM}$ potassium cyanide $(\mathrm{KCN}), 20 \mathrm{mM}$ decylubiquinone, 20mM DCIP, 50mM ATP, 0.4mM antimycin A. $15 \mu \mathrm{L}$ of homogenate was used to measure Cll activity. The negative control condition includes sodium-malonate, which inhibits CII. The final CII activity was determined integrating OD600 change over 300-800 seconds, and by subtracting the absorbance in the presence of malonate $(500 \mathrm{mM})$ from the total decrease in absorbance.

Cytochrome C Oxidase (COX/CIV) activity was determined by measuring the decrease in absorbance of cytochrome c. The rate of conversion of cytochrome $\mathrm{c}$ from a reduced to oxidized state was measured at $550 \mathrm{~nm}$ at $30^{\circ} \mathrm{C}$, in $200 \mu \mathrm{L}$ of reaction buffer $(100 \mathrm{mM}$ potassium phosphate, $\mathrm{pH} 7.5)$ containing $10 \% \mathrm{w} / \mathrm{v}$ n-dodecylmaltoside and $120 \mu \mathrm{M}$ of purified reduced cytochrome c. $6 \mathrm{uL}$ of homogenate was used to measure COX activity. The negative control condition omits tissue homogenate determine the auto-oxidation of reduced cytochrome c. The final CIV activity was determined by integrating OD550 change over150-500 seconds, and by subtracting the non-specific activity from the total decrease in absorbance.

Mitochondrial enzymatic activities were determined by averaging the duplicates. The technical variation of the duplicates was measured with and a $10 \%$ cutoff. The specific activity of each sample was calculated as the total activity minus non-specific activity (negative control), times the normalization factor. Plates were normalized by their positive controls. Because of some variation in positive controls, each plate's positive control was z-scored to the average of the positive control activity per assay. All of the activities on the plate were then multiplied by their normalization factor which is determined by $1 / z$-scored positive control.

\section{Mitochondrial DNA quantification}


mtDNA density and mtDNA copy number (mtDNAcn) were measured as previously described (77), with minor modifications. The homogenate used for the enzymatic activity measures was lysed at a 1:10 dilution in lysis buffer $(100 \mathrm{mM}$ Tris $\mathrm{HCl} \mathrm{pH} 8.5,0.5 \%$ Tween 20, and $200 \mathrm{~g} / \mathrm{ml}$ proteinase $\mathrm{K}$ ) for 10 hours at $55^{\circ} \mathrm{C}, 10$ minutes at $95^{\circ} \mathrm{C}$, and were kept at $4^{\circ} \mathrm{C}$ until used for qPCR. qPCR reactions were measured in triplicates in 384 well qPCR plates using a liquid handling station (ep-Motion5073, Eppendorf), with 12uL of master mix (TaqMan Universal Master mix fast, Life Technologies \#4444964) and 8uL of lysate. Each plate contained triplicates of a positive control (heart) and of a negative control (lysate without homogenate). qPCR reaction with Taqman chemistry was used to simultaneously quantify mitochondrial and nuclear amplicons in the same reactions: Cytochrome $c$ oxidase subunit 1 (COX1, mtDNA) and $\beta-2$ microglobulin (B2M, nDNA). The Master Mix included $300 \mathrm{nM}$ of primers and $100 \mathrm{nM}$ probe: COX1-Fwd: ACCACCATCATTTCTCCTTCTC, COX1-Rev: CTCCTGCATGGGCTAGATTT, COX1-Probe: HEX/AAGCAGGAG/ZEN/CAGGAACAGGATGAA/3IABkFQ. mB2M-Fwd: GAGAATGGGAAGCCGAACATA，mB2M-Rev: CCGTTCTTCAGCATTTGGATTT, B2M-Probe: FAM/CGTAACACA/ZEN/GTTCCACCCGCCTC/3IABkFQ.

The plate was quickly centrifuged and cycled in a QuantStudio 7 flex instrument (Applied Biosystems Cat\# 448570 ) at $50^{\circ} \mathrm{C}$ for $2 \mathrm{~min}, 95^{\circ} \mathrm{C}$ for $20 \mathrm{sec}, 95^{\circ} \mathrm{C}$ for $1 \mathrm{~min}, 60^{\circ} \mathrm{C}$ for $20 \mathrm{sec}$ for 40x cycles. To ensure comparable Ct values across plates and assays, thresholds for fluorescence detection for both mitochondrial and nuclear amplicons were set to 0.08 . Triplicates for each sample were averaged for mtDNA and nDNA, and an exclusion cutoff of Cts $>33$ was applied. For samples with triplicates C.V.S $>0.02$, the triplicates were checked, and outlier values removed where appropriate, and the remaining duplicates were used. The final cutoff was C.V. > 0.1 (10\%); and any samples with a C.V. > 0.1 were discarded. The mtDNAcn was derived from the $\Delta \mathrm{Ct}$ calculated by subtracting the average mtDNA Ct from the average 
nDNA Ct. mtDNAcn was calculated by $2^{(\Delta C t)} \times 2$. For measures of mtDNA density, the Ct value was linearized as $2^{\mathrm{Ct}} /\left(1 / 10^{-12}\right)$ to derive relative mtDNA abundance per unit of tissue.

In tissues of similar cellular density (number of cell nuclei per area or mass of tissue), mtDNAcn (mtDNA:nDNA) provides an accurate reflection of mtDNA genome density per cell. However, different brain regions vary widely in their cellularity (up tp 8.5-fold), mostly because some defined areas such as the granular layer of the cerebellum are populated with numerous small cell bodies, whereas other regions such as the molecular layer of the DG are completely acellular and devoid of cell bodies/nuclei. Nevertheless, acellular tissue compartments filled with dentrites can be rich in mitochondria and mtDNA, and therefore the number of mtDNA copies per unit of tissue $\left(\mu \mathrm{m}^{3}\right.$ or $\mathrm{mg}$ of tissue) is a more generalizable and accurate estimate of mtDNA density between brain regions.

\section{Mitochondrial Health Index}

The mitochondrial health index $(\mathrm{MHI})$ integrates the 5 primary mitochondrial features, yielding an overall score of mitochondrial respiratory chain activity on a per mitochondrion basis (21). The simple equation uses the activities of Complexes I, II, and IV as a numerator, divided by two indirect markers of mitochondrial content, citrate synthase activity and mtDNA density: $\mathrm{MHI}=(\mathrm{Cl}+\mathrm{Cll}+\mathrm{CIV}) /\left(\mathrm{CS}+\mathrm{mtDNA}_{\text {density }+1)}{ }^{*} 100\right.$. A value of 1 is added as a third factor on the denominator to balance the equation. Values for each of the 5 features are mean centered (value of an animal relative to all other animals) such than an animal with average activity for all features will have an $\mathrm{MHI}$ of $100[(1+1+1) /(1+1+1) * 100=100]$.

\section{Immunohistochemical staining}

Immunofluorescent staining was used to quantify local Succinate dehydrogenase complex, subunit A (SDHA) expression in the cerebellum. Slides with $200 \mu \mathrm{m}$ coronal sections bearing the cerebellum were taken from storage at $-80^{\circ} \mathrm{C}$ and allowed to warm up to room 
temperature for one hour. The sections were then fixed in freshly prepared $4 \%$ PFA for 5 minutes and immediately washed twice in a 0.1\% PBS-Tween 20 (PBS-Tw) solution. They then underwent dehydration through iced cold $70 \%$ methanol for $10 \mathrm{~min}, 95 \%$ methanol for $10 \mathrm{~min}$, $100 \%$ methanol for $20 \mathrm{~min}, 95 \%$ methanol for $10 \mathrm{~min}$ and $70 \%$ methanol for $10 \mathrm{~min}$ followed by two washes in PBS-Tw. In a PBS-Tw bath, sections were carefully unmounted from the slides using a razor blade and artist's paintbrush. One section containing an appropriate cerebellum region was selected from each animal and individually placed in 10\% Normal Donkey Serum (NDS) overnight at $4{ }^{\circ} \mathrm{C}$. They were then incubated in a 1:100 dilution of Rabbit anti-SDHA $1^{\circ}$ antibody in PBS overnight at $4{ }^{\circ} \mathrm{C}$ on a gentle shaker. The following day, they were washed repeatedly in PBS-Tw. Sections were then incubated in a 1:500 dilution of donkey anti-Rabbit $2^{\circ}$ conjugated with AlexaFluor 546 in NDS for two hours in a dark box at room temperature. They were then washed in PBS, incubated in $0.625 \mu \mathrm{g} / \mathrm{mL}$ 4',6-diamidino-2-phenylindole (DAPI) and washed once more. Finally, sections were mounted on Superfrost slides with Prolong Diamond mounting media and a No. 1.5 coverslip.

\section{Confocal imaging}

The immunofluorescent staining was imaged on a Leica SP8 confocal microscope equipped with Lightning super resolution software. The regions of interest were located using a Leica 20x 0.75 NA objective to determine areas of suitable staining surrounding the purkinje cell layer in the cerebellum. High-resolution images of the area were taken with a Leica $63 \times 1.40$ NA oil objective. For highest resolution, the images were formatted at 2496 × 2496 pixels, with a zoom of $2.50 \mathrm{x}$, line average of 8 , pinhole size of $0.50 \mathrm{AU}$. The final pixel size was $29.58 \mathrm{~nm} \mathrm{x}$ $29.58 \mathrm{~nm}$ and a z-stack with a step size $0.15 \mu \mathrm{m}$ was taken through the stained area. A $405 \mathrm{~nm}$ laser was used for exciting the DAPI channel with a power of $8.0 \%$ and gain of $60 \%$. A $561 \mathrm{~nm}$ laser was used for the AlexaFluor 546 channel with a power of $4.0 \%$ and a gain of $20 \%$.

\section{SDHA staining analysis}


Final images were deconvolved in the Lightning software using the default settings. Images were analyzed in ImageJ version $1.52 \mathrm{p}$. Fifty consecutive slices from each stack were selected for a section thickness of $7.5 \mu \mathrm{m}$, which roughly covered the depth of penetration of the antibodies. The 16bit images were first thresholded using the Triangle algorithm within the integrated Auto-thresholding plugin to create a binary image. The particle analyzer was then used to quantify the percent of the area stained for each slice. The percent area stained from each slice was summated to calculate the percent of volume stained. For compartmentalized data, the molecular and granular layers were separated using a rectangular selection of approximately 50px width through each stack. For the purkinje layer, a rectangle was drawn that narrowly captured the stained area of each slice. The volumetric staining for each region of interest was calculated from the percent of area stained quantified using the particle analyzer.

\section{TDA-based mapper analysis}

After creating a delta of mitochondrial features between stressed (CORT or CSDS) and control mice, the data matrix for each group was process through the TDA-based Mapper pipeline (49). The input data matrix contained 102 concatenated rows for brain regions (17 brain regions $\times 6$ mitochondrial features) and 5 or 6 columns for individual mice, based on the number of animals per group. Missing values, if any, in the input data matrix were interpolated within group using a linear interpolation. The TDA-based Mapper analysis pipeline consists of four steps. First, Mapper involves embedding the high-dimensional input data into a lower dimension $d$, using a filter function $f$. For ease of visualization, we chose $d=2$. The choice of filter function dictates what properties of the data are to be preserved in the lower dimensional space. For example, linear filter functions like classical principal component analysis (PCA) could be used to preserve the global variance of the data points in the high dimensional space. However, a large number of studies using animal models and computational research suggest that interregional interactions in the brain are multivariate and nonlinear (78-80). Thus, to better capture 
the intrinsic geometry of the data, a nonlinear filter function based on neighborhood embedding was used (49). The second step of Mapper performs overlapping n-dimensional binning to allow for compression and reducing the effect of noisy data points. Here, we divided the data into lower dimensional space into 64 bins with $70 \%$ overlap. Similar results were observed for different number of bins (e.g., 49, and 81). Third, partial clustering within each bin is performed, where the original high dimensional information is used for coalescing (or separating) data points into nodes in the low-dimensional space. Partial clustering allows to recover the loss of information incurred due to dimensional reduction in step one (81). Lastly, to generate a graphical representation of the shape of the data, nodes from different bins are connected if any data points are shared between them.

The Mapper-generated graphs can be annotated (or colored) using meta-information that was not used to construct the graphs. Here, we annotated these graphs using region-labels to examine whether mitochondrial features were similarity expressed across all regions or whether regional specificity was observed across the two groups. To quantify the extent of segregation (high regional specificity) or integration (low regional specificity) across the six mitochondrial features, we used a graph theoretical measurement of participation coefficient (51). Participation coefficient $P_{i}$ of a node $i$ is defined as:

$$
P_{i}=1-\sum_{s=1}^{N_{M}}\left(\frac{\kappa_{i s}}{k_{i}}\right)^{2}
$$

where $\kappa_{i s}$ is the number of links of node $i$ to nodes in community $s, k_{i}$ is the total degree of node $i$ and $N_{M}$ is the number of communities. The $P_{i}$ of a node $i$ is close to 1 if its links are uniformly distributed among all communities of the graph (and hence integrated) and it is close to 0 if its links are mostly within its own community (and hence segregated).

\section{Multi-slice community detection}


One of the most commonly studied mesoscale aspect of a graph is modularity, where highly modular graphs consist of cohesive groups of nodes (or communities) that are more strongly connected to each other than they are to the rest of the network (82). Examination of modularity has been recently extended to multi-slice networks that are defined by coupling multiple adjacency matrices across time or modality (55). Here, we used six slices, each derived from one of the mitochondrial features, where each slice represented weighted adjacency matrix of pair-wise Pearson's correlation between brain regions. We use categorical multi-slice community detection algorithm with the presence of all-to-all identity arcs between slices (55). The generalized modularity function for multi-slice community detection is given by

$$
Q_{\text {multislice }}=\frac{1}{2 \mu} \sum_{i j s r}\left[\left(A_{i j s}-\gamma_{s} \frac{k_{i s} k_{j s}}{2 m_{s}}\right) \delta_{s r}+\delta_{i j} C_{j s r}\right] \delta\left(g_{i s}, g_{j r}\right)
$$

Where $A_{i j s}$ represents weighted adjacencies between nodes $i$ and $j$ for each slice $s$, with interslice couplings $C_{j r s}$ that connect node $j$ in slice $r$ to itself in slice $s . \gamma_{s}$ represents resolution parameter in each slice; higher value of $\gamma_{s}$ (e.g., $\left.>1\right)$ detects smaller modules, while lower values (e.g., between 0 and 1) detects bigger modules. In line with previous work, here, we set the resolution parameter across slices to be unity, i.e., $\gamma_{s}=1$ (83). For simplicity, and as done previously, the interslice coupling parameters were also kept same across slices (55). Here, we used $C_{j s r}=0.1$. Perturbation of interslice coupling values around 0.1 produced similar results.

To estimate the stability of identified communities across the six mitochondrial slices, under the assumption that stable communities could represent convergence across mitochondrial features, we estimated module allegiance matrix (84). The module allegiance matrix summarizes the stability of community structure across slices, such that each entry $i, j$ of the matrix corresponds to the percentage of slices in which regions $i$ and $j$ belong to the same community. Finally, an iterative consensus community detection algorithm was run on the module allegiance matrix to define the large-scale networks that are convergent across slices 
defined by mitochondrial features. The iterative community detection (85) was run multiple times $(1,000)$, followed by consensus clustering to get stable results for identifying large-scale networks of brain regions (86).

\section{Comparison of mito-based networks with transcriptomic- and structural connectivity-}

\section{based networks}

To compare our mitochondrial communities against other modalities, we examined the organization of gene co-expression in the mouse brain as well as mouse structural connectome data. We utilized the Allen Brain Atlas' ISH (in situ hybridization) feature, which maps each gene onto a reference standard coordinate atlas image, providing a spatial estimate of transcript levels representing gene expression (56). Specifically, we used the Anatomic Gene Expression Atlas (AGEA) (https://mouse.brain-map.org/agea), which is a a three-dimensional male adult C57BL/6J mouse brain atlas based on the ISH gene expression images. The AGEA feature allows for selecting both a 'seed voxel' and a target 'selected voxel' from exact brain coordinates coordinates, yielding the transcriptome-wide correlation between the seed and target regions. The correlation is a measure of the average co-expression between the two voxels. The co-expression values were obtained for all possible pair of brain regions (17x17 matrix), yielding a gene co-expression correlation matrix to which the structure of our mitobased networks could be compared using a permutation text.

The structural connectome data was obtained from The Allen Mouse Brain Connectivity Atlas (57), which is a mesoscale connectome of the adult mouse brain. We utilized the normalized projection strength values for the all brain regions of interest. Because this atlas does not distinguish between dorsal and ventral dentate gyrus, data was obtained for 16 brain regions. For three brain regions where the Atlas provides connectivity values for multiple subregions (OFC, VN, Cerebellum), the connectivity values were averaged across the 
subregions to yield a global measure for each region, thus matching the dimensionality of our mitochondrial dataset.

\section{Statistical analysis}

Standardized effect sizes (Hedge's g) were computed to quantify the effect of stress conditions on mitochondrial features. Significant effect sizes were determined by the $95 \%$ confidence intervals. Two-way ANOVAs were used to compare the effects of the two stressors on the mitochondrial measures across all tissues, and to compare the average mitochondrialbehavior correlations between brain regions and tissues for all behaviors. A binomial test was used to determine if the proportion of brain regions exhibiting an increase or decrease in mitochondrial features differed from chance level (50\%). Correlations between behavioral Zscores and mitochondrial activities were estimated using Spearman's $r(r)$ to guard against inflation. Frequency distributions of the effect sizes for the two stressors and of the mitochondrial-behavior correlations were fitted with Gaussian curves and analyzed by one sample t tests compared to the null distribution. Correlations between tissues' mitochondrial activities were measured as Pearson's r. A survival curve using Mantel-Cox log-rank test was generated for novelty suppressed feeding test latencies because the test is capped at $600 \mathrm{sec}$. Hierarchical clustering was performed on the data using Euclidian distance with Ward's clustering algorithm. Statistical analyses were performed with Prism 9 (GraphPad) and Metaboanalyst version 4 (87).

\section{Funding}

Work of the authors is supported by GM119793, MH122706, MH108719, MH090964, $\mathrm{MH} 111918, \mathrm{MH} 119735$. 


\section{Acknowledgements}

The authors are grateful to David Sulzer and René Hen for their support, and Amira Millette for her technical assistance.

\section{Author contributions}

M.P., C.A., D.D. conceived the project. A.R., P.R., C.A. collected the data. A.R., P.R., A.W.L., analyzed the data. A.R., M.S., P.R. generated the figures. A.R., M.S., C.S., E.V.M., D.D., C.A., M.P. interpreted the data. A.R. and M.P. drafted the manuscript. All authors reviewed and edited the final version of the manuscript.

\section{Competing Interests}

The authors declare no competing interest related to this work. MP has consulted and received funding from Epirium Bio.

\section{References}

1. K. Friston, The free-energy principle: a unified brain theory? Nat Rev Neurosci 11, $127-$ 138 (2010).

2. C. N. Hall, M. C. Klein-Flugge, C. Howarth, D. Attwell, Oxidative phosphorylation, not glycolysis, powers presynaptic and postsynaptic mechanisms underlying brain information processing. J Neurosci 32, 8940-8951 (2012).

3. J. Courchet et al., Terminal axon branching is regulated by the LKB1-NUAK1 kinase pathway via presynaptic mitochondrial capture. Cell 153, 1510-1525 (2013).

4. E. Gebara et al., Mitofusin-2 in the Nucleus Accumbens Regulates Anxiety and Depression-like Behaviors Through Mitochondrial and Neuronal Actions. Biol Psychiatry 89, 1033-1044 (2021). 
5. A. V. Lechuga-Vieco et al., Cell identity and nucleo-mitochondrial genetic context modulate OXPHOS performance and determine somatic heteroplasmy dynamics. Sci Adv 6, eaba5345 (2020).

6. B. Ugur et al., The Krebs Cycle Enzyme Isocitrate Dehydrogenase 3A Couples Mitochondrial Metabolism to Synaptic Transmission. Cell Rep 21, 3794-3806 (2017).

7. S. K. Kwon et al., LKB1 Regulates Mitochondria-Dependent Presynaptic Calcium Clearance and Neurotransmitter Release Properties at Excitatory Synapses along Cortical Axons. PLoS Biol 14, e1002516 (2016).

8. T. Sun, H. Qiao, P. Y. Pan, Y. Chen, Z. H. Sheng, Motile axonal mitochondria contribute to the variability of presynaptic strength. Cell Rep 4, 413-419 (2013).

9. M. Khacho, R. Harris, R. S. Slack, Mitochondria as central regulators of neural stem cell fate and cognitive function. Nat Rev Neurosci 20, 34-48 (2019).

10. A. U. Joshi et al., Fragmented mitochondria released from microglia trigger A1 astrocytic response and propagate inflammatory neurodegeneration. Nat Neurosci 22, 1635-1648 (2019).

11. D. Tomasi, G. J. Wang, N. D. Volkow, Energetic cost of brain functional connectivity. Proc Natl Acad Sci U S A 110, 13642-13647 (2013).

12. K. Mann, S. Deny, S. Ganguli, T. R. Clandinin, Coupling of activity, metabolism and behaviour across the Drosophila brain. Nature, (2021).

13. M. Picard, B. S. McEwen, E. S. Epel, C. Sandi, An energetic view of stress: Focus on mitochondria. Front Neuroendocrinol 49, 72-85 (2018).

14. C. Fecher et al., Cell-type-specific profiling of brain mitochondria reveals functional and molecular diversity. Nat Neurosci 22, 1731-1742 (2019).

15. M. Picard, R. T. Hepple, Y. Burelle, Mitochondrial functional specialization in glycolytic and oxidative muscle fibers: tailoring the organelle for optimal function. Am J Physiol Cell Physiol 302, C629-641 (2012).

16. S. Rath et al., MitoCarta3.0: an updated mitochondrial proteome now with sub-organelle localization and pathway annotations. Nucleic Acids Res 49, D1541-D1547 (2021).

17. C. Menacho, A. Prigione, Tackling mitochondrial diversity in brain function: from animal models to human brain organoids. Int J Biochem Cell Biol 123, 105760 (2020). 
18. V. Riedl et al., Metabolic connectivity mapping reveals effective connectivity in the resting human brain. Proc Natl Acad Sci U S A 113, 428-433 (2016).

19. H. Ju, D. S. Bassett, Dynamic representations in networked neural systems. Nat Neurosci 23, 908-917 (2020).

20. P. A. Kragel, L. Koban, L. F. Barrett, T. D. Wager, Representation, Pattern Information, and Brain Signatures: From Neurons to Neuroimaging. Neuron 99, 257-273 (2018).

21. M. Picard et al., A Mitochondrial Health Index Sensitive to Mood and Caregiving Stress. Biol Psychiatry 84, 9-17 (2018).

22. B. Buwalda et al., Long-term effects of social stress on brain and behavior: a focus on hippocampal functioning. Neurosci Biobehav Rev 29, 83-97 (2005).

23. C. Anacker et al., Neuroanatomic Differences Associated With Stress Susceptibility and Resilience. Biol Psychiatry 79, 840-849 (2016).

24. R. C. Bagot et al., Circuit-wide Transcriptional Profiling Reveals Brain Region-Specific Gene Networks Regulating Depression Susceptibility. Neuron 90, 969-983 (2016).

25. B. Myers, J. M. McKlveen, J. P. Herman, Glucocorticoid actions on synapses, circuits, and behavior: implications for the energetics of stress. Front Neuroendocrinol 35, 180196 (2014).

26. J. D. Gray, T. G. Rubin, R. G. Hunter, B. S. McEwen, Hippocampal gene expression changes underlying stress sensitization and recovery. Mol Psychiatry 19, 1171-1178 (2014).

27. B. Labonte et al., Sex-specific transcriptional signatures in human depression. Nat Med 23, 1102-1111 (2017).

28. A. M. Magarinos, J. M. Verdugo, B. S. McEwen, Chronic stress alters synaptic terminal structure in hippocampus. Proc Natl Acad Sci U S A 94, 14002-14008 (1997).

29. F. Hollis et al., Mitochondrial function in the brain links anxiety with social subordination. Proc Natl Acad Sci U S A 112, 15486-15491 (2015).

30. X. Xie et al., Depression-like behaviors are accompanied by disrupted mitochondrial energy metabolism in chronic corticosterone-induced mice. J Steroid Biochem Mol Biol 200, 105607 (2020).

31. V. N. Babenko, D. A. Smagin, A. G. Galyamina, I. L. Kovalenko, N. N. Kudryavtseva, Altered Slc25 family gene expression as markers of mitochondrial dysfunction in brain 
regions under experimental mixed anxiety/depression-like disorder. BMC Neurosci 19, 79 (2018).

32. M. Weger et al., Mitochondrial gene signature in the prefrontal cortex for differential susceptibility to chronic stress. Sci Rep 10, 18308 (2020).

33. M. Picard, B. S. McEwen, Psychological Stress and Mitochondria: A Systematic Review. Psychosom Med 80, 141-153 (2018).

34. Y. Hara et al., Presynaptic mitochondrial morphology in monkey prefrontal cortex correlates with working memory and is improved with estrogen treatment. Proc Natl Acad Sci U S A 111, 486-491 (2014).

35. M. S. Sharpley et al., Heteroplasmy of mouse mtDNA is genetically unstable and results in altered behavior and cognition. Cell 151, 333-343 (2012).

36. M. A. van der Kooij et al., Diazepam actions in the VTA enhance social dominance and mitochondrial function in the nucleus accumbens by activation of dopamine D1 receptors. Mol Psychiatry 23, 569-578 (2018).

37. A. K. Kanellopoulos et al., Aralar Sequesters GABA into Hyperactive Mitochondria, Causing Social Behavior Deficits. Cell 180, 1178-1197 e1120 (2020).

38. T. Kasahara et al., Mice with neuron-specific accumulation of mitochondrial DNA mutations show mood disorder-like phenotypes. Mol Psychiatry 11, 577-593, 523 (2006).

39. L. Holper, D. Ben-Shachar, J. J. Mann, Multivariate meta-analyses of mitochondrial complex I and IV in major depressive disorder, bipolar disorder, schizophrenia, Alzheimer disease, and Parkinson disease. Neuropsychopharmacology 44, 837-849 (2019).

40. A. Strasser et al., Glutamine-to-glutamate ratio in the nucleus accumbens predicts effortbased motivated performance in humans. Neuropsychopharmacology 45, 2048-2057 (2020).

41. A. Strasser, L. Xin, R. Gruetter, C. Sandi, Nucleus accumbens neurochemistry in human anxiety: A 7 T (1)H-MRS study. Eur Neuropsychopharmacol 29, 365-375 (2019).

42. A. Cherix et al., Metabolic signature in nucleus accumbens for anti-depressant-like effects of acetyl-L-carnitine. Elife 9, (2020). 
43. F. J. Miller, F. L. Rosenfeldt, C. Zhang, A. W. Linnane, P. Nagley, Precise determination of mitochondrial DNA copy number in human skeletal and cardiac muscle by a PCRbased assay: lack of change of copy number with age. Nucleic Acids Res 31, e61 (2003).

44. A. Stepanova, Y. Shurubor, F. Valsecchi, G. Manfredi, A. Galkin, Differential susceptibility of mitochondrial complex II to inhibition by oxaloacetate in brain and heart. Biochim Biophys Acta 1857, 1561-1568 (2016).

45. D. J. David et al., Neurogenesis-dependent and -independent effects of fluoxetine in an animal model of anxiety/depression. Neuron 62, 479-493 (2009).

46. S. A. Golden, H. E. Covington, 3rd, O. Berton, S. J. Russo, A standardized protocol for repeated social defeat stress in mice. Nat Protoc 6, 1183-1191 (2011).

47. C. Anacker et al., Hippocampal neurogenesis confers stress resilience by inhibiting the ventral dentate gyrus. Nature 559, 98-102 (2018).

48. V. Krishnan et al., Molecular adaptations underlying susceptibility and resistance to social defeat in brain reward regions. Cell 131, 391-404 (2007).

49. M. Saggar et al., Towards a new approach to reveal dynamical organization of the brain using topological data analysis. Nat Commun 9, 1399 (2018).

50. G. Singh, F. Memoli, G. Carlsson. (The Eurographics Association, Eurographics Symposium on Point-Based Graphics, 2007 ).

51. R. Guimera, L. A. Nunes Amaral, Functional cartography of complex metabolic networks. Nature 433, 895-900 (2005).

52. M. D. Greicius, B. Krasnow, A. L. Reiss, V. Menon, Functional connectivity in the resting brain: a network analysis of the default mode hypothesis. Proc Natl Acad Sci U S A 100, 253-258 (2003).

53. J. S. Damoiseaux et al., Consistent resting-state networks across healthy subjects. Proc Natl Acad Sci U S A 103, 13848-13853 (2006).

54. S. M. Smith et al., Correspondence of the brain's functional architecture during activation and rest. Proc Natl Acad Sci U S A 106, 13040-13045 (2009).

55. P. J. Mucha, T. Richardson, K. Macon, M. A. Porter, J. P. Onnela, Community structure in time-dependent, multiscale, and multiplex networks. Science 328, 876-878 (2010).

56. E. S. Lein et al., Genome-wide atlas of gene expression in the adult mouse brain. Nature 445, 168-176 (2007). 
57. S. W. Oh et al., A mesoscale connectome of the mouse brain. Nature 508, 207-214 (2014).

58. J. Richiardi et al., BRAIN NETWORKS. Correlated gene expression supports synchronous activity in brain networks. Science 348, 1241-1244 (2015).

59. M. E. Newman, Modularity and community structure in networks. Proc Natl Acad Sci U $S$ A 103, 8577-8582 (2006).

60. S. Tonegawa, X. Liu, S. Ramirez, R. Redondo, Memory Engram Cells Have Come of Age. Neuron 87, 918-931 (2015).

61. P. A. Kragel, K. S. LaBar, Decoding the Nature of Emotion in the Brain. Trends Cogn Sci 20, 444-455 (2016).

62. D. J. Morgan et al., Glucocorticoid receptor isoforms direct distinct mitochondrial programs to regulate ATP production. Scientific reports 6, 26419 (2016).

63. S. H. Yang et al., Mitochondrial localization of estrogen receptor beta. Proc Natl Acad Sci U S A 101, 4130-4135 (2004).

64. J. V. Haxby et al., Distributed and overlapping representations of faces and objects in ventral temporal cortex. Science 293, 2425-2430 (2001).

65. C. W. Woo et al., Separate neural representations for physical pain and social rejection. Nat Commun 5, 5380 (2014).

66. S. Fuke, M. Kubota-Sakashita, T. Kasahara, Y. Shigeyoshi, T. Kato, Regional variation in mitochondrial DNA copy number in mouse brain. Biochim Biophys Acta 1807, 270274 (2011).

67. T. Cayci, Y. G. Kurt, E. O. Akgul, B. Kurt, Does mtDNA copy number mean mitochondrial abundance? J Assist Reprod Genet 29, 855 (2012).

68. R. J. Longchamps et al., Evaluation of mitochondrial DNA copy number estimation techniques. PLoS One 15, e0228166 (2020).

69. M. Wachsmuth, A. Hubner, M. Li, B. Madea, M. Stoneking, Age-Related and Heteroplasmy-Related Variation in Human mtDNA Copy Number. PLoS Genet 12, e1005939 (2016).

70. P. Bohne, M. K. Schwarz, S. Herlitze, M. D. Mark, A New Projection From the Deep Cerebellar Nuclei to the Hippocampus. Front Neural Circuits 13, 51 (2019). 
71. B. B. Sandrew, C. E. Poletti, Limbic influence on the periaqueductal gray: a single unit study in the awake squirrel monkey. Brain Res 303, 77-86 (1984).

72. S. S. Maisonnette, M. C. Kawasaki, N. C. Coimbra, M. L. Brandão, Effects of lesions of amygdaloid nuclei and substantia nigra on aversive responses induced by electrical stimulation of the inferior colliculus. Brain Res Bull 40, 93-98 (1996).

73. S. A. Golden, H. E. Covington, O. Berton, S. J. Russo, A standardized protocol for repeated social defeat stress in mice. Nat Protoc 6, 1183-1191 (2011).

74. C. Anacker et al., Behavioral and neurobiological effects of GnRH agonist treatment in mice-potential implications for puberty suppression in transgender individuals. Neuropsychopharmacology 46, 882-890 (2021).

75. R. Bakker, P. Tiesinga, R. Kotter, The Scalable Brain Atlas: Instant Web-Based Access to Public Brain Atlases and Related Content. Neuroinformatics 13, 353-366 (2015).

76. J. P. S. Boutaleb, C. Hindorf, A. Pelegrin, J. Barbet, P. Kotzki, M. Bardiès Impact of Mouse Model on Preclinical Dosimetry in Targeted Radionuclide Therapy. Proceedings of the IEEE 97, 2076-2085 (2009).

77. M. Picard et al., Mitochondrial dysfunction and lipid accumulation in the human diaphragm during mechanical ventilation. Am J Respir Crit Care Med 186, 1140-1149 (2012).

78. S. Anzellotti ，E. Fedorenko ，A. J. E. Kell, A. Caramazza ，R. Saxe Measuring and Modeling Nonlinear Interactions Between Brain Regions with fMRI. bioRxiv 074856, (2017).

79. J. J. DiCarlo, D. Zoccolan, N. C. Rust, How does the brain solve visual object recognition? Neuron 73, 415-434 (2012).

80. M. Riesenhuber, T. Poggio, Hierarchical models of object recognition in cortex. Nat Neurosci 2, 1019-1025 (1999).

81. C. Geniesse, O. Sporns, G. Petri, M. Saggar, Generating dynamical neuroimaging spatiotemporal representations (DyNeuSR) using topological data analysis. Netw Neurosci 3, 763-778 (2019).

82. M. Girvan, M. E. Newman, Community structure in social and biological networks. Proc Natl Acad Sci U S A 99, 7821-7826 (2002). 
83. D. S. Bassett et al., Dynamic reconfiguration of human brain networks during learning. Proc Natl Acad Sci U S A 108, 7641-7646 (2011).

84. D. S. Bassett, M. Yang, N. F. Wymbs, S. T. Grafton, Learning-induced autonomy of sensorimotor systems. Nat Neurosci 18, 744-751 (2015).

85. L. G. S. Jeub, M. Bazzi, I. S. Julta, P. J. Mucha. (2011-2019).

86. A. Lancichinetti, S. Fortunato, Consensus clustering in complex networks. Sci Rep 2, 336 (2012).

87. J. Chong, J. Xia, Using MetaboAnalyst 4.0 for Metabolomics Data Analysis, Interpretation, and Integration with Other Omics Data. Methods Mol Biol 2104, 337-360 (2020). 


\section{Figure legends}

Figure 1. Behavioral and neuroendocrine stressors enhance the diversity of mitochondrial phenotypes across brain regions. (A) Effect of CORT and CSDS on mitochondrial features across brain regions and peripheral tissues relative to controls. Effect sizes are quantified as Hedge's g, with significant effect sizes (95\% confidence interval) labeled with the fold difference. $P$ value from 2-way ANOVA. (B) Pairwise comparisons between brain regions from $(A)$, colored by $p$-value $(-\log 10)$. (C) Gaussian fit for the frequency distribution of the effect sizes in $A$ on all 6 mitochondrial features in all 17 brain regions ( $n=102$ pairs); onesample $t$ test against null hypothesis $g=0$. (D) Number of brain regions in which mitochondrial features are either higher or lower in CORT and CSDS mice relative to controls; ${ }^{*} \mathrm{P}<0.05$, ${ }^{* * *} P<0.001$ from a binomial test. (E) Exemplar representations of Mapper input and output. The pie-chart based annotation of graph nodes allowed us to examine the degree of co-regulation of mito-features across brain regions. (F) Topological data analysis (TDA) based Mapper approach to determine if brain regions were co-regulated in their stress-induced mitochondrial recalibrations for the two groups. (G) Graph-theoretical measure of participation coefficient, measuring the degree of segregation/integration among brain regions in each graph, indicating that CORT-induced mitochondrial recalibrations were more region-specific (or segregated) whereas CSDS caused a more integrated response.

Figure 2. Association patterns for brain-wide mitochondrial function and mouse behavior. (A) Mitochondrial phenotyping and behavioral profiling of inter-individual variation in a heterogenous population of mice; OFT, open field test; EPM, elevated plus maze; NSF, novelty suppressed feeding; SI, social interaction test. (B) Gaussian fits of the frequency distributions for all correlations $(n=102)$ between the composite mitochondrial health index $(\mathrm{MHI})$ and each behavioral test; one sample t test against null hypothesis $r=0$ (other mitochondrial features are shown in Extended Data Fig. 8). (C) Individual correlations for the 17 brain region, across the 6 mitochondrial features, for each behavioral score (see Extended Data Figure 9 for additional details) quantified as Spearman's $r$. The strongest correlations for each behavioral test are plotted below. An adjusted p-value of $<0.002$ was applied (false-discovery rate $1 \%$ ). All tests have been adjusted so that a higher score indicates higher anxiety-like behavior (see Methods for details). (D) Average correlation of each behavior for the brain (B) and tissue (T) mitochondrial features; 2-way ANOVA.

Figure 3. Mitochondrial function-based connectivity across anatomical brain regions. (A) Connectivity matrix of mitochondrial features across brain regions, using all 6 mitochondrial features across the animal cohort $(n=27)$. The matrix is ordered by hierarchical clustering (Euclidian distance, Ward's clustering). (B) Cross-correlation of each mitochondrial feature to the other 5 measures within each brain region ( $n=17$ brain regions). (C) Global connectivity based on the average correlation for each brain region with all other regions. (D) Average correlation of mitochondrial features between brain region's $(B)$, peripheral tissues ( $T$ ), and between brain regions and tissues (B-T); $<<0.0001$, Ordinary one-way ANOVA with multiple comparisons. (E) Multi-slice community detection analysis on mitochondrial measures across the 17 brain regions, with mitochondrial features represented in six separate layers, resulting in 
bioRxiv preprint doi: https://doi.org/10.1101/2021.06.02.446767; this version posted June 3, 2021. The copyright holder for this preprint (which was not certified by peer review) is the author/funder, who has granted bioRxiv a license to display the preprint in perpetuity. It is made available under aCC-BY 4.0 International license.

(F) three distinct communities or brain networks. Modular structure confirmed by permutation test, $\mathrm{p}<0.0001)$. (G) Average mito-behavior correlation by module for each behavioral test (top). The bottom panel shows networks with each region color-coded by its average correlation with behaviors; for OFT and EPM (left) and SI (right). Modularity metrics anchored in whole-brain transcriptome and the structural connectome data are shown in Extended Data Fig. 10.

Table 1. Expanded abbreviations and Bregma coordinates. All regions were taken bilaterally except those marked with *, which were obtained by collecting tissue from two consecutive slices 
A

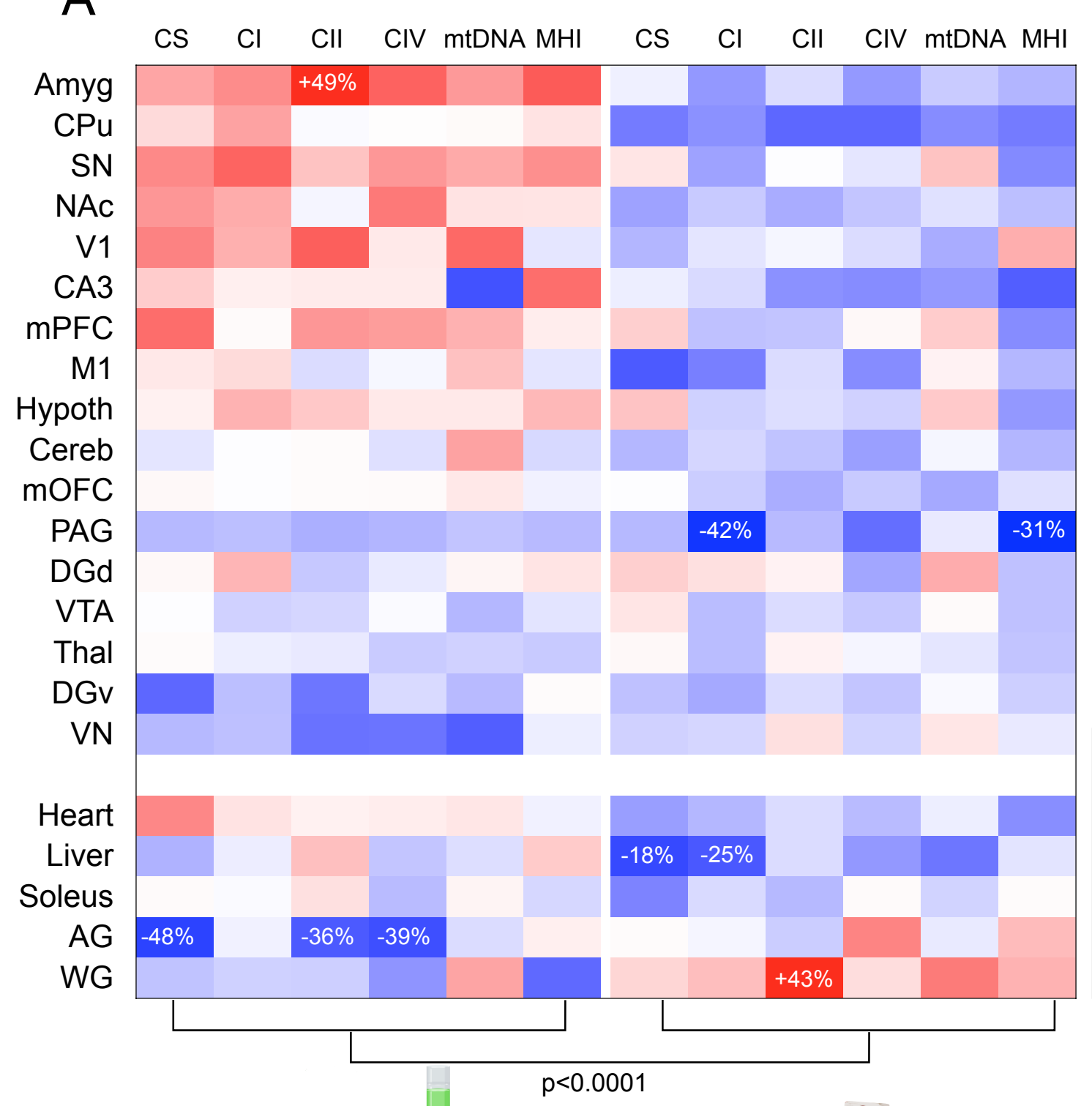

B

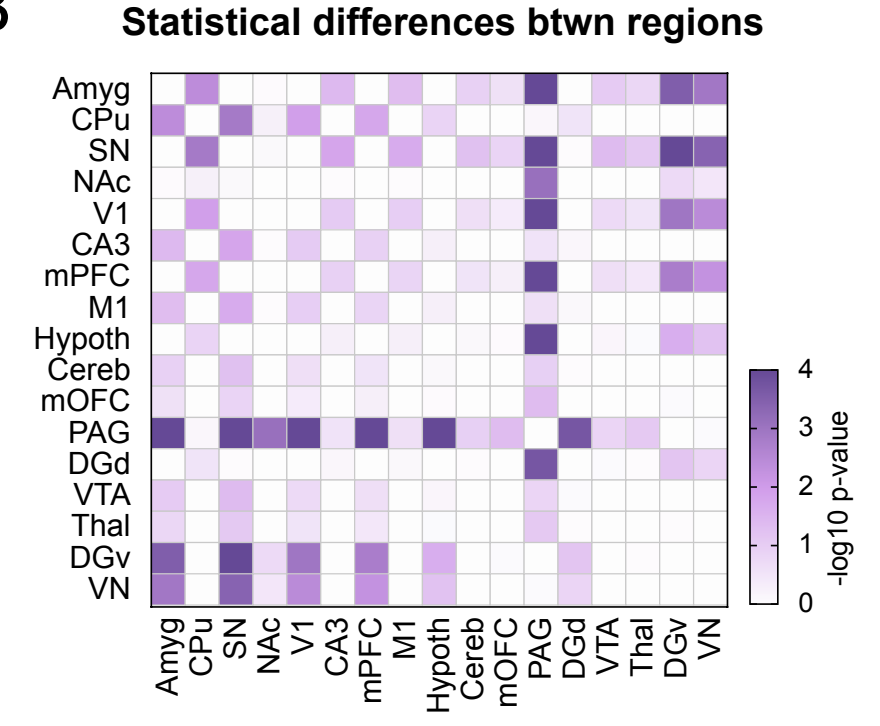

C Global effects of stressors

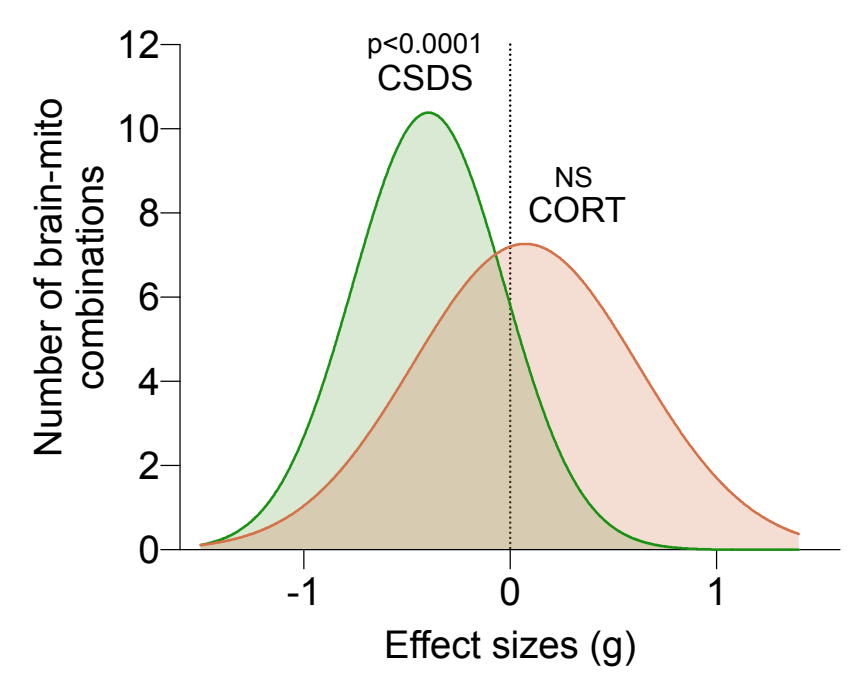

D Direction of mitochondrial responses to stressors

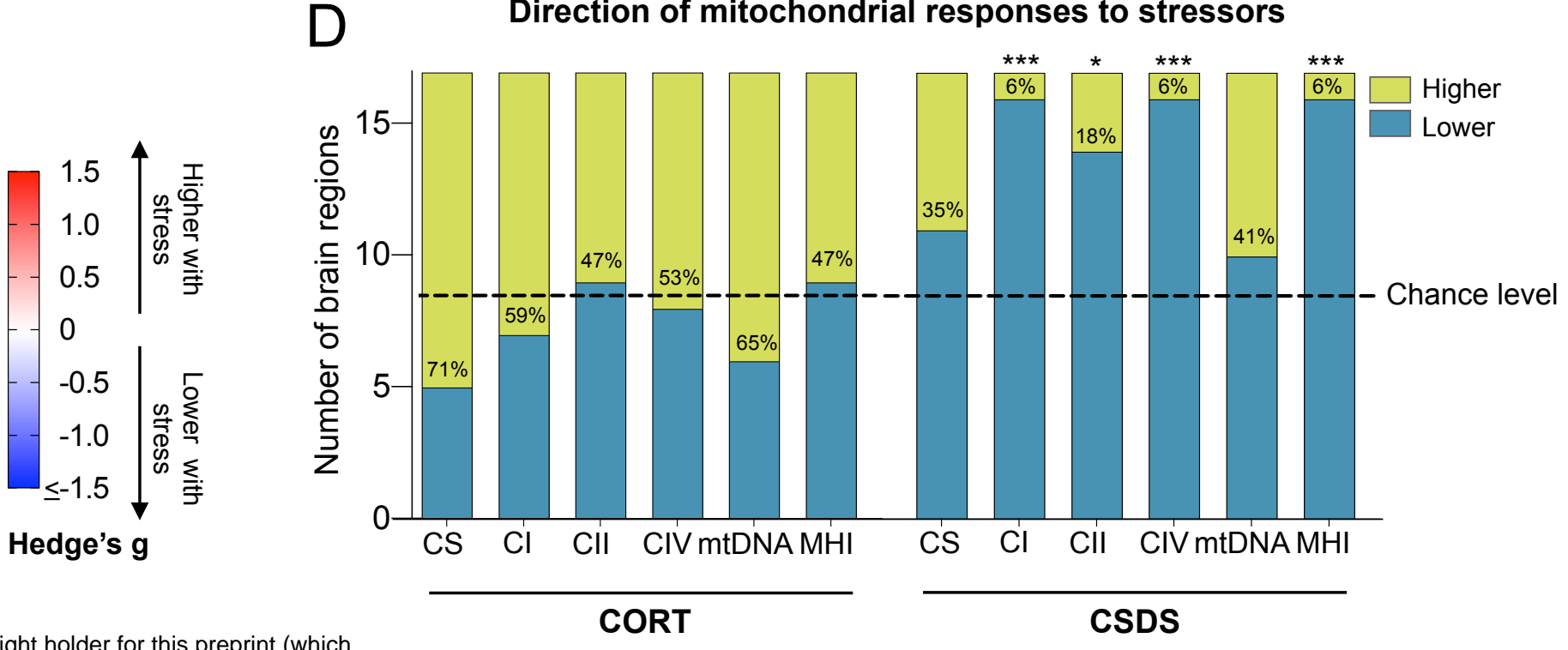

bioRxiv preprint doi: https://doi org/10.1101/2021.06.02.446767; this version posted June 3, 2021. The copyright holder for this preprint (which was not certified by peer review) is the author/funder, who has granted bioBxiva a license to display the preprint in perpetuity. It is made CORT CSDS

$\mathrm{E}$

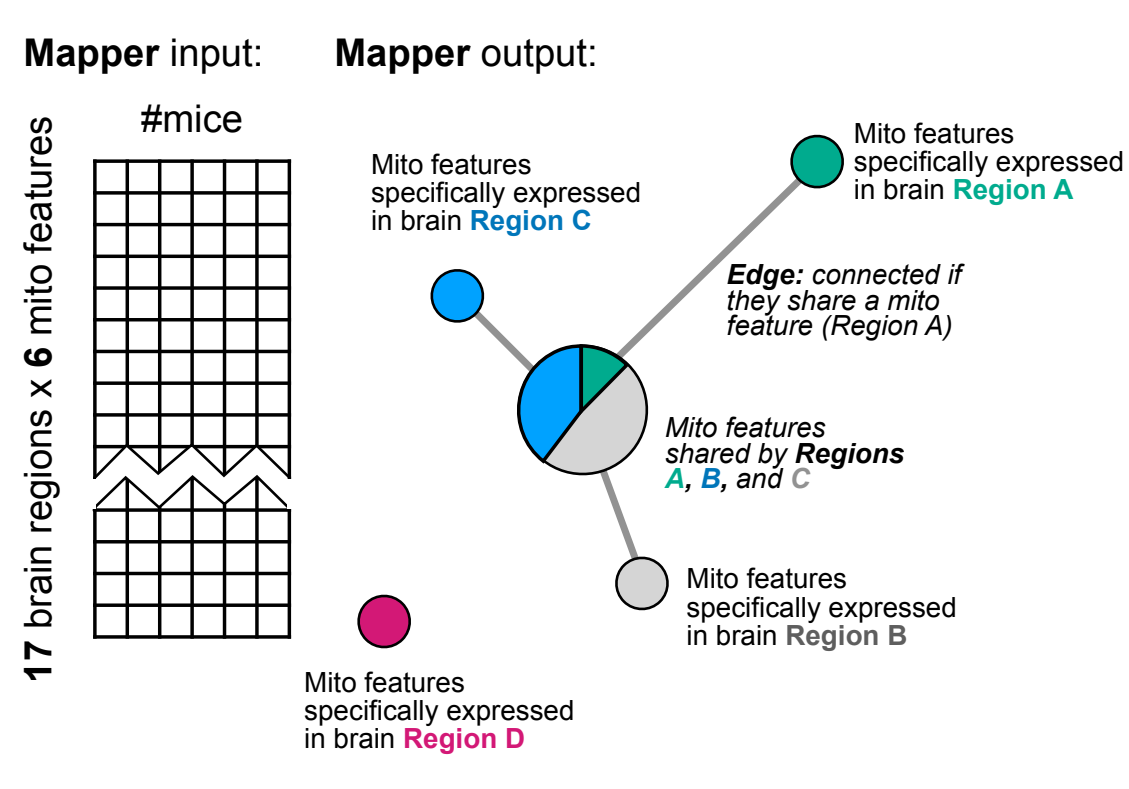

G

Degree of segregation vs integration

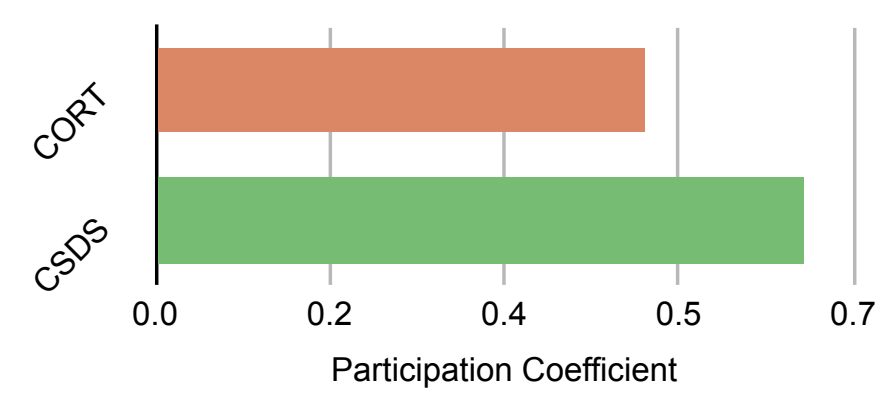

$\mathrm{F}$

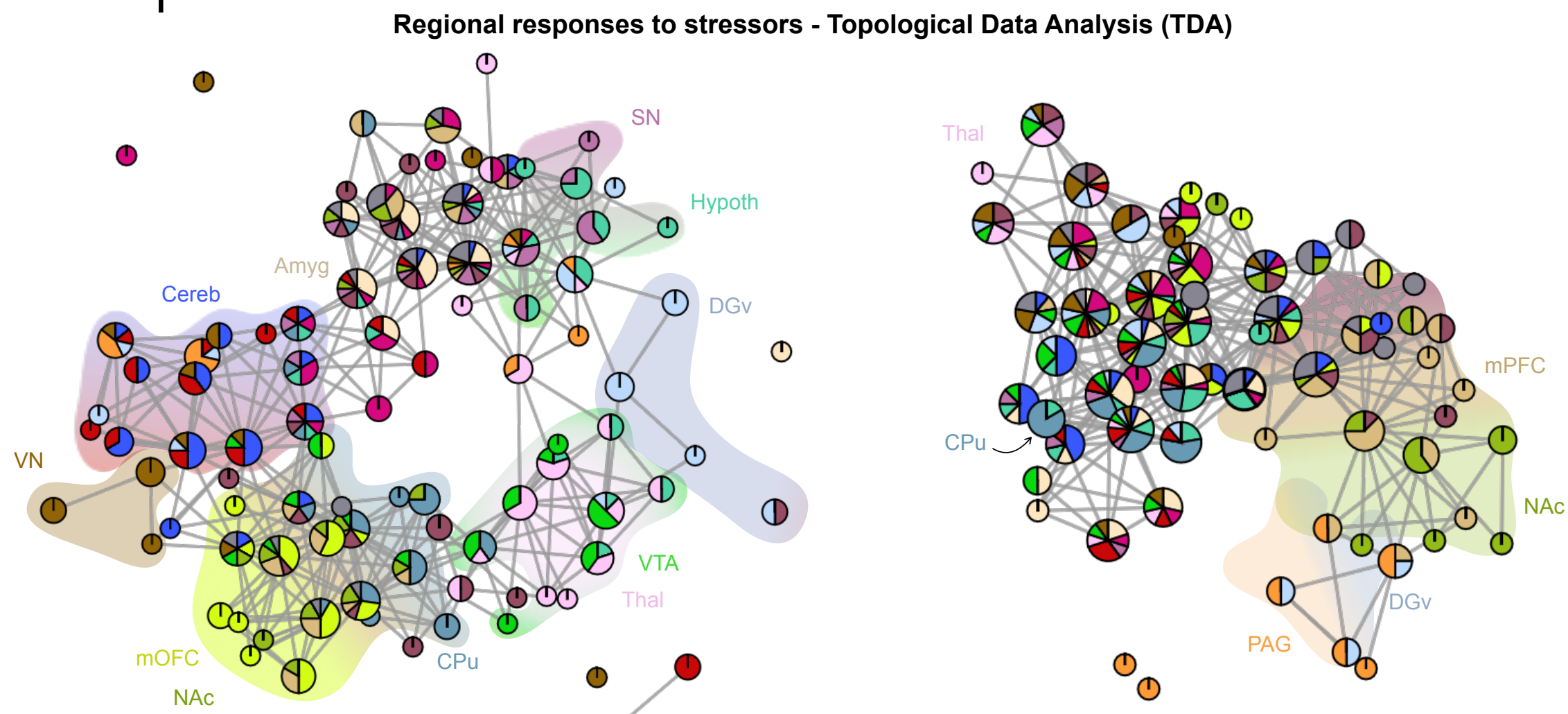

CSDS

CORT

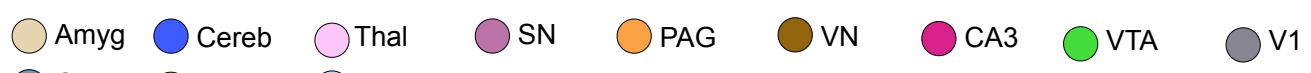


A

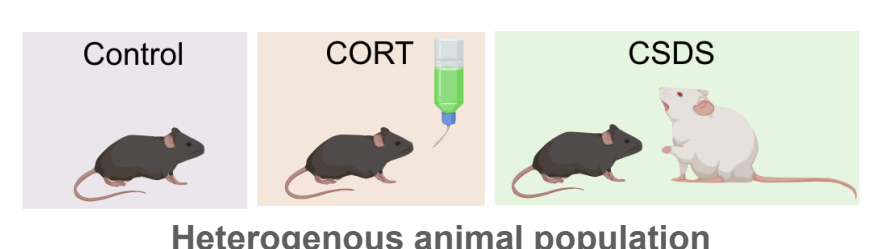

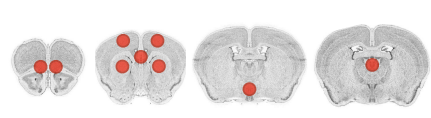

(100)

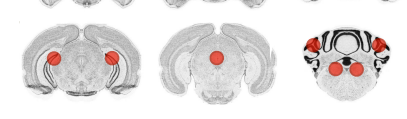

17 brain regions

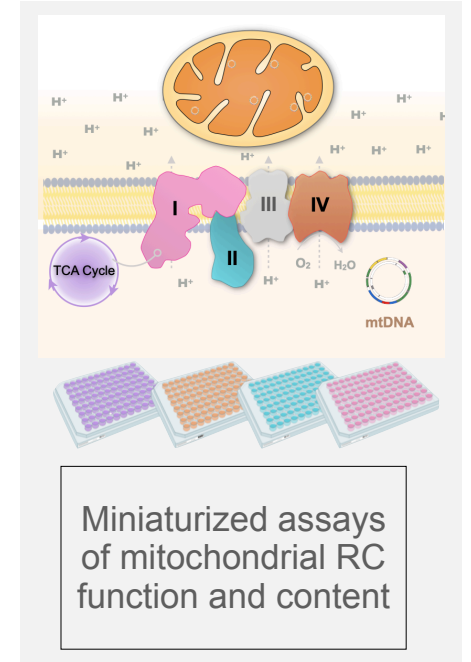

Diversity of

mitochondrial health

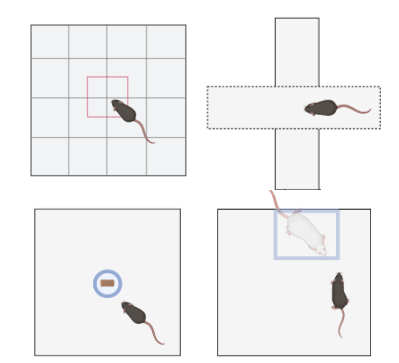

Behavioral Tests

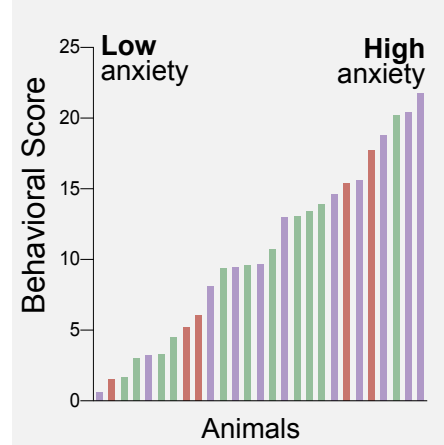

$$
\begin{aligned}
& 4 \text { anxiety-related } \\
& \text { behavioral tests }
\end{aligned}
$$
Diversity of

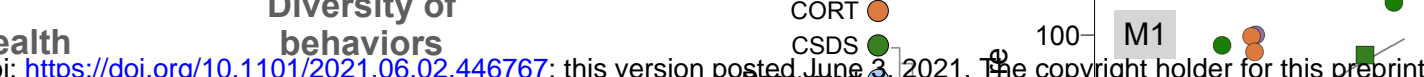

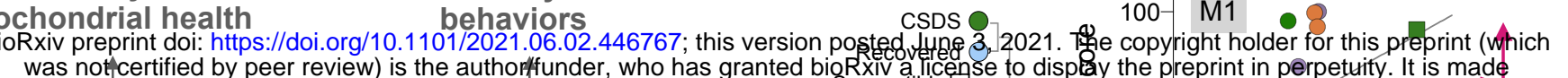
Correlation?
B

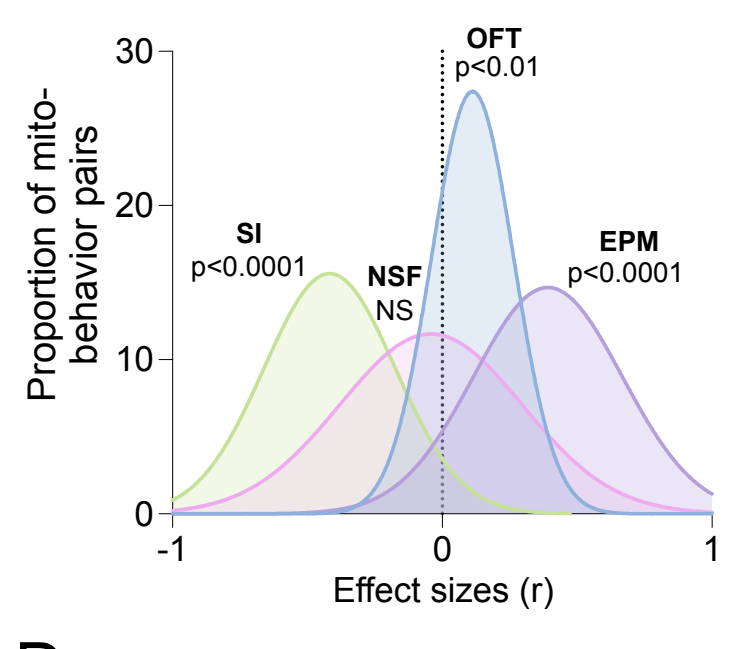

D $<<0.0001$

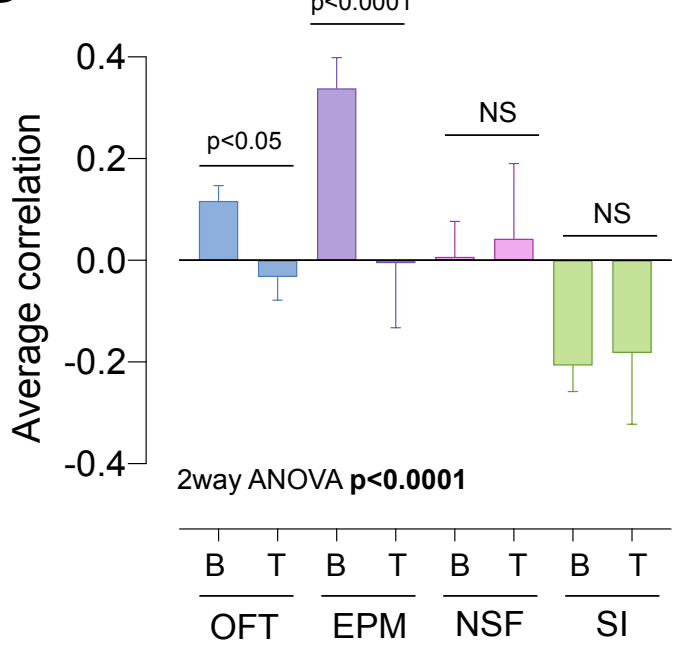

C
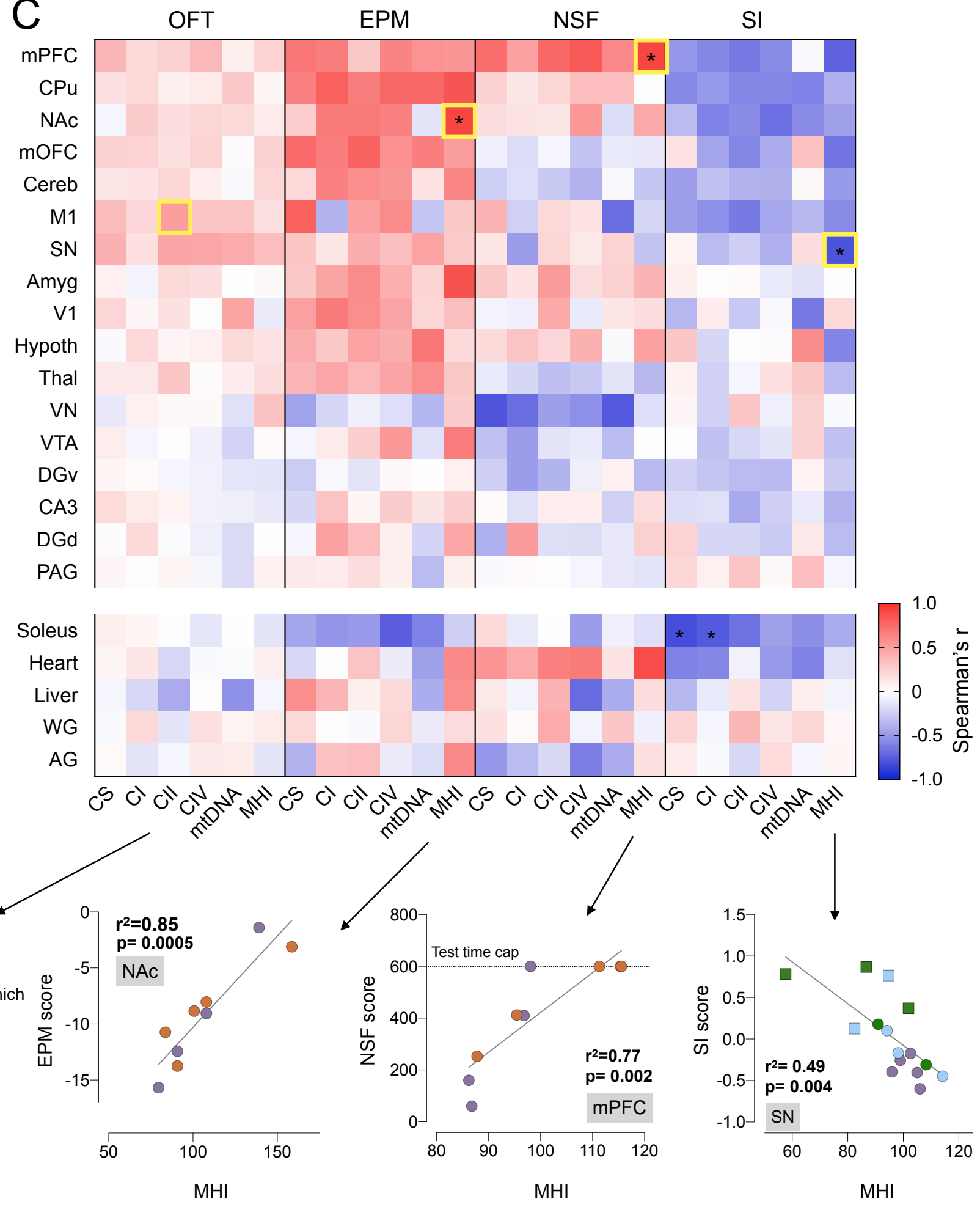
A

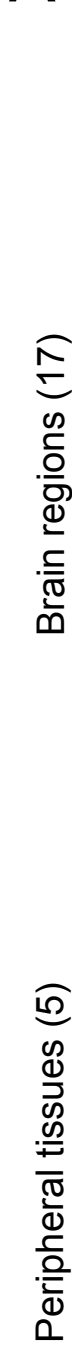

Connectivity matrix based on mitochondrial features

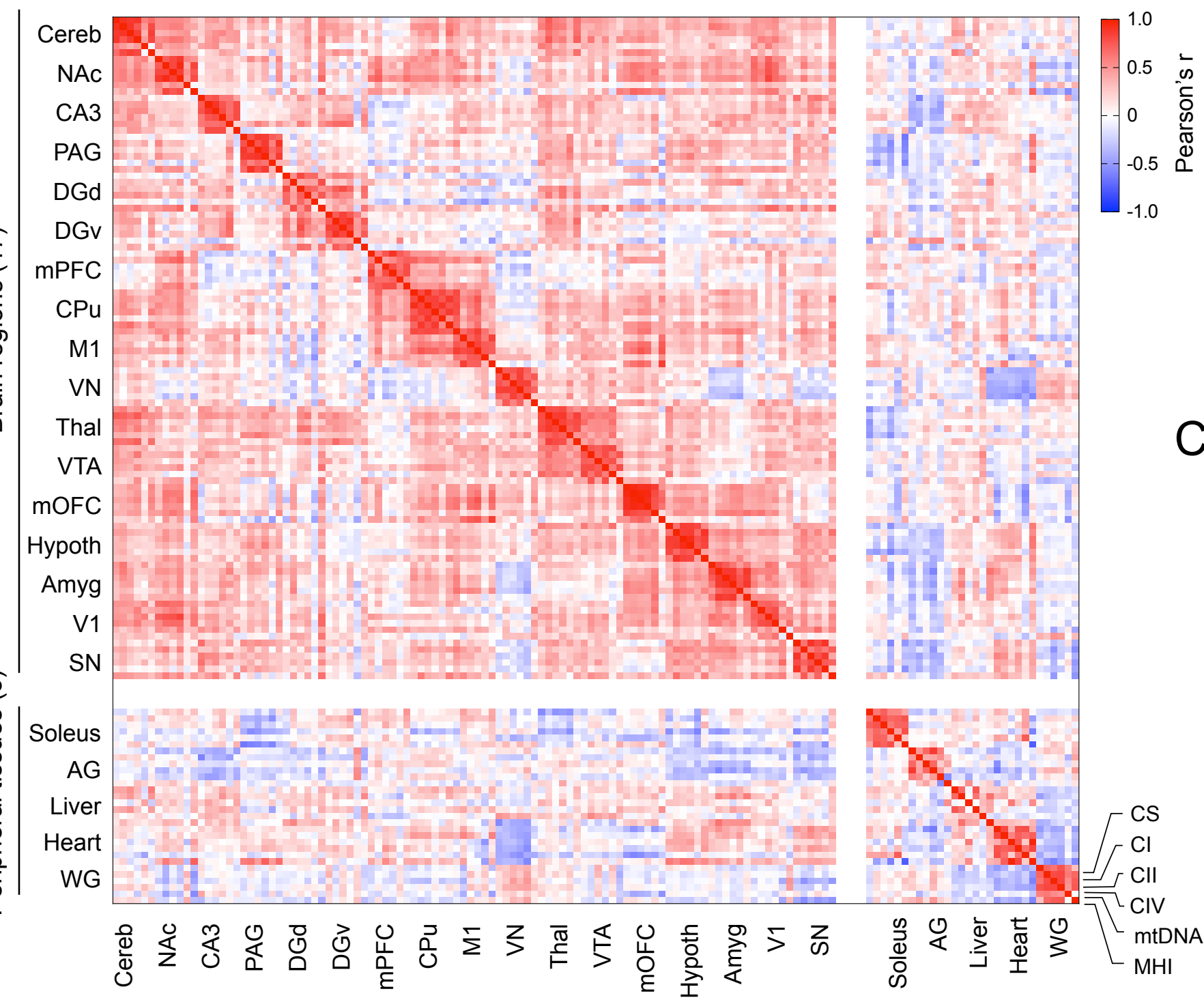

B Connectivity by mito feature

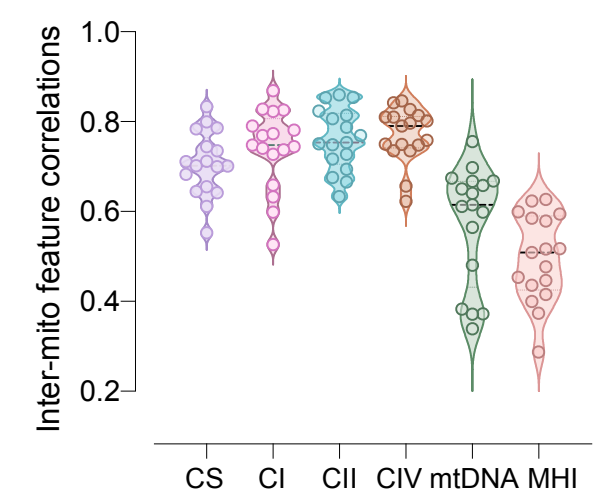

C

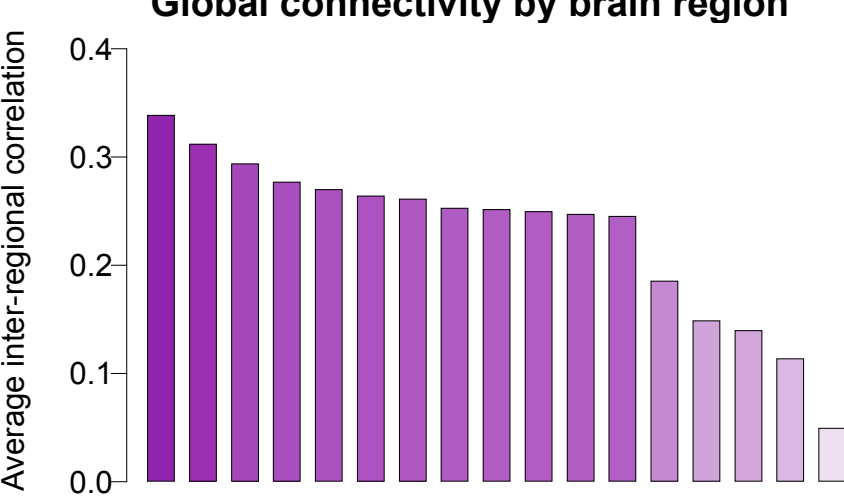

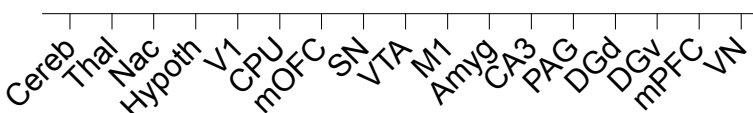

Brain vs tissues

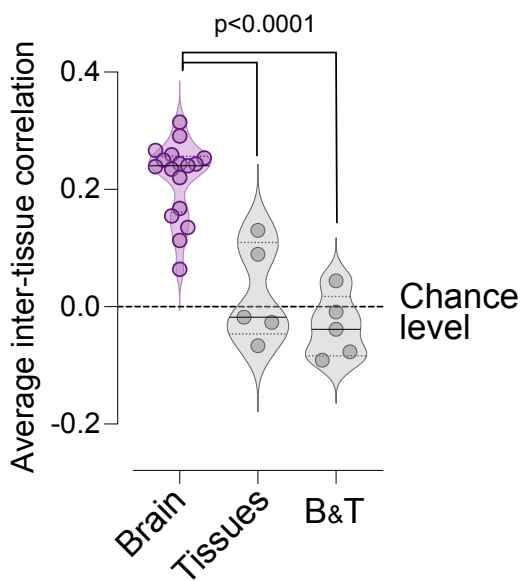

$\mathrm{E}$

Mitochondria-driven community detection among brain regions

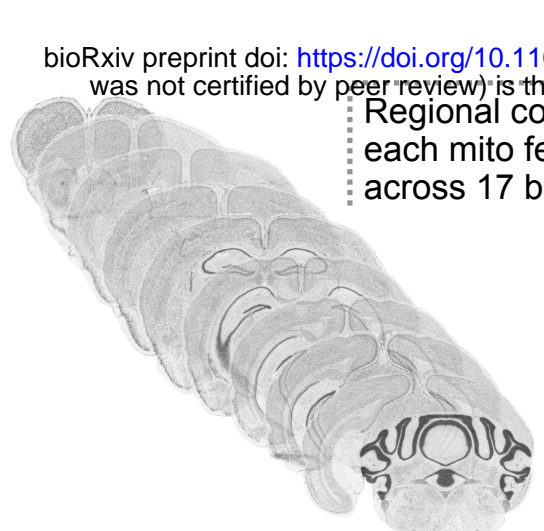
each mito feature egions

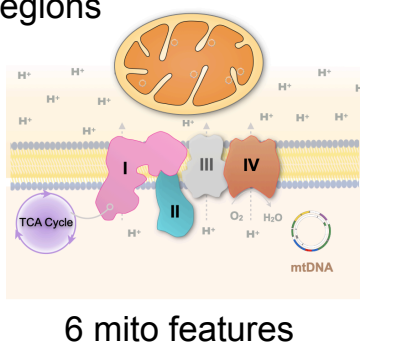

Salience/

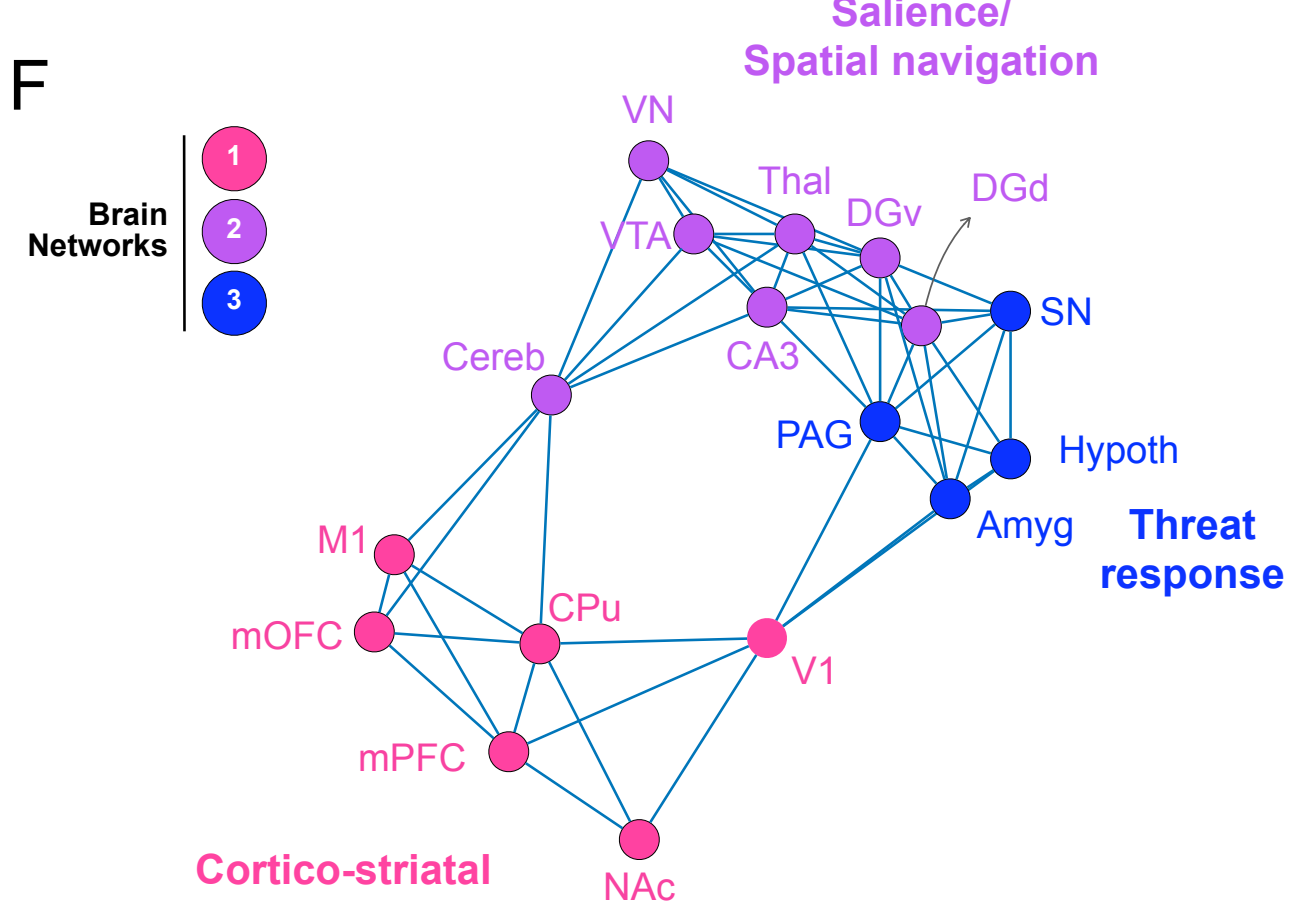

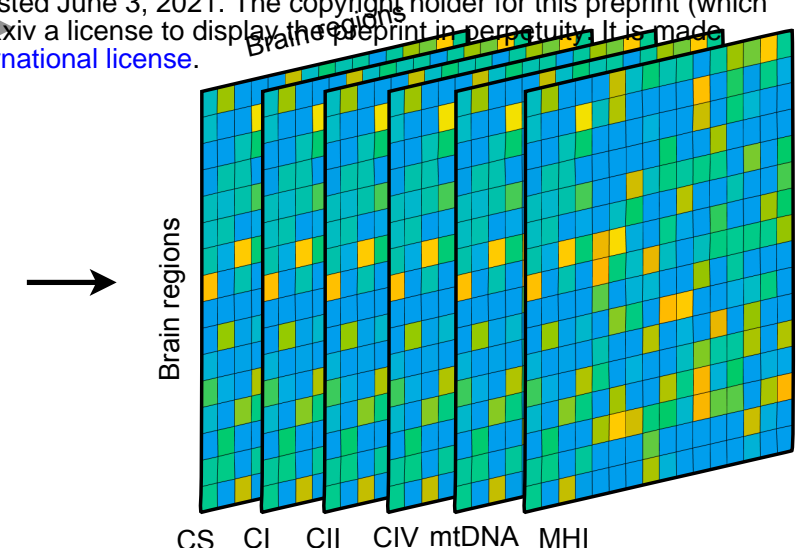

Categorical Multi-slice community detection

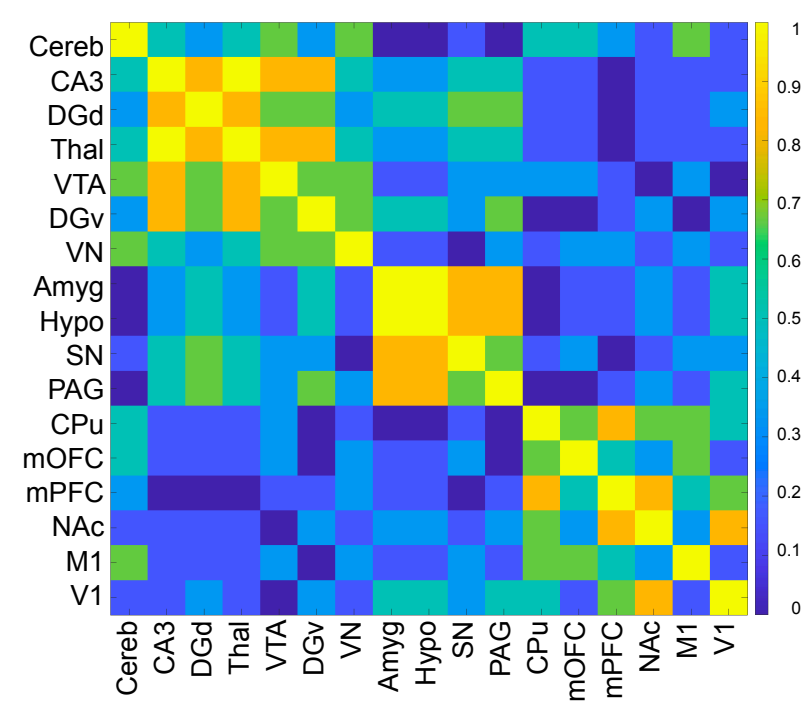

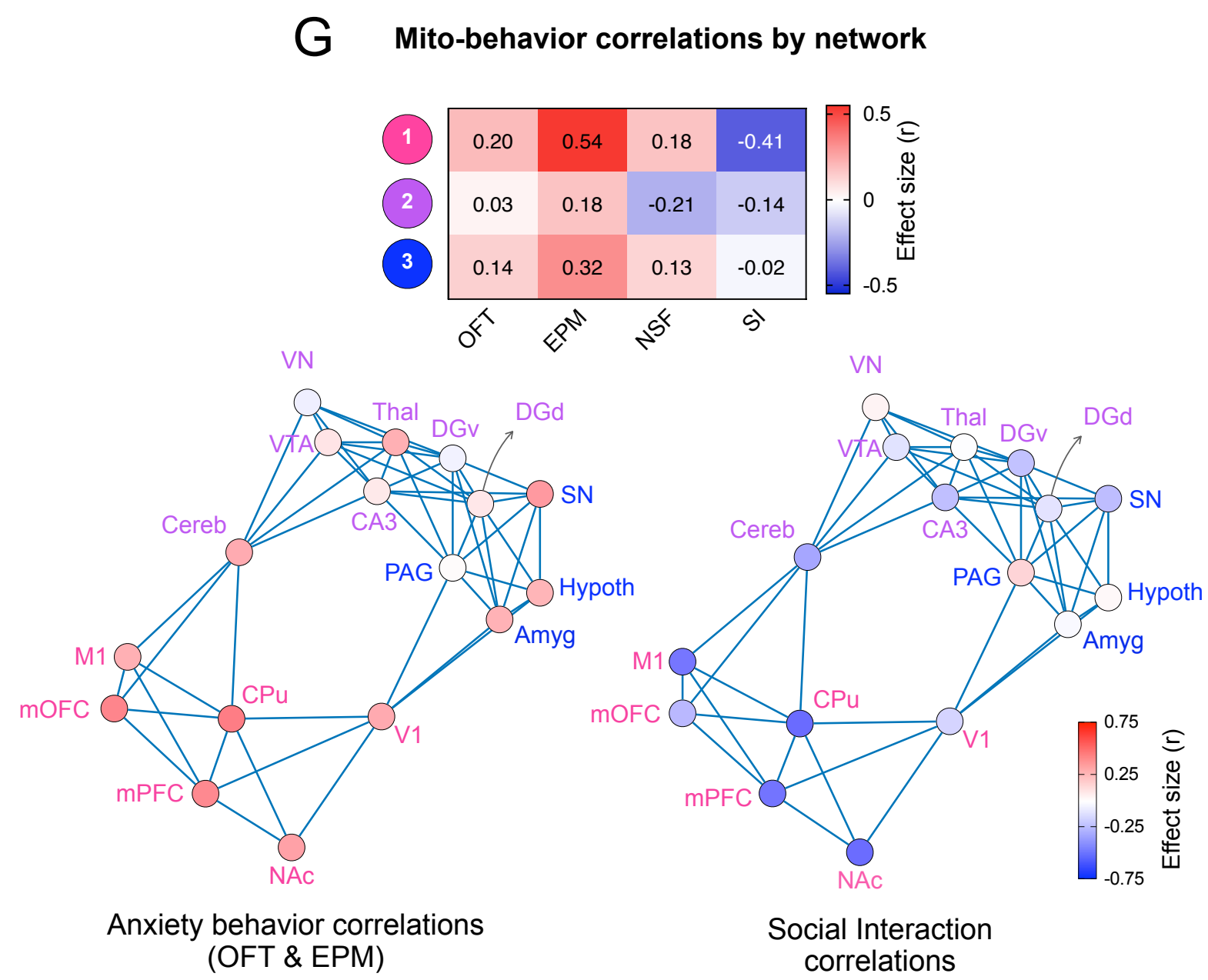


bioRxiv preprint doi: https://doi.org/10.1101/2021.06.02.446767; this version posted June 3, 2021. The copyright holder for this preprint (which was not certified by peer review) is the author/funder, who has granted bioRxiv a license to display the preprint in perpetuity. It is made available under aCC-BY 4.0 International license.

\begin{tabular}{|c|c|c|}
\hline Abbreviation & Region name & Bregma / Anatomical location \\
\hline mOFC & Medial orbitofrontal cortex & 2.42 \\
\hline mPFC & Medial prefrontal cortex & 1.22 \\
\hline $\mathrm{CPu}$ & Caudoputamen & 1.22 \\
\hline NAc & Nucleus accumbens & 1.22 \\
\hline M1 & Primary motor cortex & 1.22 \\
\hline Hypoth & Hypothalamus & $-0.48^{*}$ \\
\hline Thal & Thalamus & $-0.68^{*}$ \\
\hline DGd & Dorsal dentate gyrus & -1.78 \\
\hline Amyg & Amygdala & -1.78 \\
\hline CA3 & CA3 region & -2.58 \\
\hline VTA & Ventral tegmental area & -2.58 \\
\hline V1 & Primary visual cortex & -2.58 \\
\hline SN & Substantia nigra & -3.08 \\
\hline DGv & Ventral dentate gyrus & -3.38 \\
\hline PAG & Periaqueductal grey & $-3.78^{*}$ \\
\hline Cereb & Cerebellum & -6.38 \\
\hline VN & Vestibular nuclei & -6.38 \\
\hline$A G$ & Adrenal glands & Both glands pooled \\
\hline Liver & Liver & Inferior portion of left lobe \\
\hline Heart & Heart & Inner wall of left ventricle \\
\hline Soleus & Red oxidative skeletal muscle & Tendon-to-tendon, right leg \\
\hline WG & White glycolytic skeletal muscle & Superficial medial head, right leg \\
\hline
\end{tabular}

